\title{
Very-near-field dynamics in the injection of two-dimensional gas jets and thin liquid sheets between two parallel high-speed gas streams
}

\author{
By ENRIQUE LÓPEZ-PAGÉS, CÉSAR DOPAZO† \\ AND NORBERTO FUEYO \\ LITEC-CSIC/Universidad de Zaragoza, María de Luna 3, 50018, Zaragoza, Spain
}

(Received 13 August 2001 and in revised form 17 March 2004)

A numerical investigation of the velocity, pressure and vorticity fields very near the injection of flat and thin two-dimensional gas jets or liquid sheets between two parallel high-speed gas coflows is performed. The motivation of this research is to uncover some basic physical mechanisms underlying twin-fluid atomization. Conservation equations and boundary and initial conditions are presented for both single-phase jets and two-phase liquid sheet/gas-stream systems. Both infinitely thin and thick solid walls are considered. Apart from the gas Strouhal and Reynolds numbers appearing in the dimensionless single-phase flow equations, the liquid Reynolds number, the momentum flux ratio, the gas/liquid velocity ratio and the Weber number enter the two-phase flow dimensionless formulation. The classical numerical techniques for single-phase jets are supplemented with the volume-of-fluid (VOF) method for interface tracking and the continuum surface force (CSF) method to include surface tension in two-phase flow systems. Ad hoc convection algorithms in combination with a developed version of the fractional-step scheme allows a significant reduction of the numerical diffusion, maintaining localized and sharp interfaces. The action of the surface tension is correctly found via the CSF with a smoothed scalar-field approximation.

Results for single-phase jets with thin-wall injectors indicate qualitatively correct features and trends when varying the Reynolds number and the coflow/jet ratios: thick-wall injectors significantly modify the vorticity and pressure near fields; increasing the Reynolds number leads to larger flow disturbances; larger coflow/jet velocity ratios yield more perturbed near flow fields. For single-phase jets the Strouhal number as a function of the Reynolds number follows the usual trends of flows behind a circular cylinder.

For two-phase flows, increasing the gas Reynolds number leads to larger liquidsheet deformations and to a reduction of the breakup length; a plot of the gas Strouhal number, in the presence of a liquid sheet, as a function of the gas Reynolds number displays a monotonically decreasing curve, contrary to that for a gas jet. This observation strongly suggests that the gas vortex shedding mechanism is modified by the liquid-sheet motion. The gas vortex shedding frequency as a function of the liquid-sheet oscillation frequency follows a straight line with a slope of approximately $45^{\circ}$ for momentum flux ratios greater than roughly 0.45 ; for values below 0.45 the gas vortex shedding frequency remains constant while the liquid sheet varies its oscillation frequency. Increasing the surface tension leads to a larger breakup length. 
Thin trailing edges almost double the sheet oscillation frequency and more than halve the perturbation wavelength compared to thick trailing edges.

\section{Introduction}

Twin-fluid atomizers are commonplace in aircraft gas turbines and some thermoelectric power plants. Although phenomenological rules provide reasonable bases for design, the underlying physical mechanisms for the liquid sheet deformation and breakup under the action of the gas streams as well as the liquid/gas dynamic interactions are, to a large extent, not well understood.

The following historical review will solely allude to large-aspect-ratio thin flat liquid sheet geometries. Water bells generated by impinging liquid jets on a disk were first experimentally studied by Savart (1833) and later taken up by Taylor (1959). Pioneering linear stability analyses of thin inviscid liquid sheets were performed by Squire (1953) for a quiescent air stream and York, Stubbs \& Tek (1953) for two inviscid air coflows. Hagerty \& Shea (1955) experimentally observed sinusoidal and varicose modes using an oscillating nozzle.

Water fans were extensively investigated by Dombrowski and co-workers in the 1970s. For example, Crapper, Dombrowski \& Jepson (1975) considered viscous liquid and air with a slightly parabolic liquid velocity profile and an exponentially decaying air velocity profile (quiescent air at infinity) for the basic flows; inclusion of viscosity, apparently, widened the frequency instability range and for large liquid velocities no maxima for the growth rate curve as a function of wavenumber for any Weber number appeared. Air vortices were visualized and the influence of the vortex-induced pressure field on the growth rate of the sheet perturbations was hinted at Clark \& Dombrowski (1972) conducted the first nonlinear instability analysis for a liquid sheet via a perturbation expansion technique.

Detailed flow visualization and measurements on large-aspect-ratio liquid sheets with two high-speed air coflows were performed by Stapper \& Samuelsen (1990), Stapper, Sowa \& Samuelsen (1992) and Mansour \& Chigier (1990, 1991). A close correspondence between the spanwise/streamwise liquid filaments in these flows and the spanwise/streamwise vorticity structures previously unveiled in a single-phase shear layer (Lasheras \& Choi 1988; Meiburg \& Lasheras 1988) seemed evident; the sheet breakup process was qualitatively described as changing from a 'cellular' type to a 'stretched streamwise ligament' mode as the air/water velocity ratio increased. The sheet oscillation frequency was found to depend linearly on the air velocity and to be only a weak function of the liquid velocity. Increasing the velocity ratio caused the sinusoidal mode to dominate the varicose one. The influence of the surface tension and the liquid viscosity on the breakup characteristic length and time scales and, thus, on the mean drop diameter was considered by, among others, Stapper et al. (1992); to a large extent, the basic mechanisms are, however, independent of these fluid properties. Lozano et al. (1996) and Vich, Dumouchel \& Ledoux (1996) recently paid special attention to the sheet instability development and growth in the very near field behind the injection nozzle tip. Barreras (1998) performed detailed visualizations of both the liquid sheet and the vortex systems of the adjacent air coflows as well as extensive measurements for varying liquid and air velocities using laser-induced fluorescence, concluding that the air flow dynamics has a direct effect on the liquid sheet motion. Recently, Berthoumieu \& Carentz (2000) have re-examined Barreras' 
experiment. The equivalent axisymmetric system has been investigated by, among others, Lasheras \& Hopfinger (2000), Lasheras, Villermaux \& Hopfinger (1998) and Raynal (1997).

Most instability studies have dealt with the linear analysis of a liquid sheet moving in quiescent air at infinity. Lin, Lian \& Creighton (1990) found that the inclusion of gas viscosity destabilizes the sheet and decreases the wavelengths of both the varicose and the sinuous modes. The temporal growth of a perturbed viscous liquid sheet in an inviscid gas was investigated by Li \& Tankin (1991) and a viscosity-enhanced instability uncovered. A temporal growth analysis of the previous configuration in two and three dimensions was performed by Ibrahim (1994) and Ibrahim \& Akpan (1996). Two developed viscous-flow-type parabolic velocity profiles for the basic state of the liquid and the quiescent gas were employed by Teng, Lin \& Chen (1997); absolute instability takes place for Weber numbers close to unity. Linear theory implies no changes of the sheet thickness during the growth of the sinusoidal mode and, thus, cannot be expected to predict liquid sheet breakup. Kim \& Sirignano (2000) integrated the inviscid conservation equations across the sheet thickness, treating it as an independent variable; they found that nonlinear effects are stronger when the spanwise and streamwise wavenumbers are close and they computed the sheet thickness evolution in terms of those wavenumbers and the Weber number. Jazayeri \& Li (2000) presented an inviscid nonlinear instability analysis for a liquid sheet in a gas medium at rest; sheet thickness variations leading to breakup result from the superposition of the fundamental sinuous mode on the first harmonic (the varicose one); the breakup characteristic time and length scales are found to decrease as the initial disturbance amplitude increases and as the Weber number and the gas/liquid density ratio grow.

Conditions relevant to twin-fluid atomization, i.e. thin liquid sheets sandwiched between two high-speed air coflows have only been investigated in the last decade using both linear and nonlinear instability analysis. Rangel \& Sirignano (1991) conducted inviscid two-dimensional linear and nonlinear studies; a vortex discretization method was adapted for the nonlinear case, finding the disturbance growth rate to be a function of the sheet thickness, the wavelength and the gas/liquid density ratio. For small values of the latter the sinusoidal mode dominates, while for values greater than $1 / 4$ the varicose mode takes over. The vortex discretization method was extended to three-dimensional nonlinear inviscid instability studies by Lozano, García-Olivares \& Dopazo (1998), obtaining the sheet-thickness time evolution both in the downstream and the spanwise directions; some indication of streamwise liquid filament formation was presented. Yang (1992), Ibrahim (1994) and Cousin \& Dumouchel (1996) undertook inviscid linear studies for gas/liquid velocity ratios greater than one; Ibrahim (1995) considered air compressibility. Li (1994) analysed the instability of a viscous liquid sheet between two gas streams of unequal velocities. The para-sinuous and the para-varicose modes, resembling but different from the classical sinuous and varicose modes, were introduced; the values of the Weber number and of the liquid and gas velocities control the two modes. Two parabolic velocity profiles for the liquid and the gas basic flows were fitted, using a KármánPohlhausen-type method, by Hauke et al. (2001) to investigate the sinusoidal mode growth rate of a liquid sheet with two high-speed air coflows; the liquid and gas Reynolds numbers, the air/liquid momentum flux ratio, the Weber number, the air/liquid velocity ratio and the ratio of a boundary layer characteristic thickness to sheet thickness emerged as the relevant parameters of this linear stability study. Lozano et al. (2001) compared these results with their experimental data. 
Compared to single-phase numerical solutions, interface tracking and the treatment of surface tension are the two main additional problems to address when modelling and computing gas/liquid flows with evolving interfaces (Scardovelli \& Zaleski 1999). Localizing the interfaces, applying correctly the stress balances at them and computing their evolution can be achieved using either moving grids (Hirt, Amsdem \& Cook 1974; Fritts \& Boris 1979; Harten \& Hyman 1983; Hyman 1983, 1984; Ryskin \& Leal 1984; Cristini, Blawzdziewics \& Lowenberg 1998) or fixed ones (Hirt \& Nichols 1981; Lötstedt 1982; Ashgriz \& Poo 1991; Lafaurie et al. 1994; Mashayek \& Ashgriz 1995; Zaleski 1996; Chen et al. 1999). Additionally, scalar markers (Hirt \& Nichols 1981; Zaleski 1996) as well as particle tracers (Welch et al. 1966; Nichols \& Hirt 1975; Glimm, Marchesin \& McBryan 1981) can be employed; while Welch et al. (1966) distribute the particles within the whole volume to be traced, Nichols \& Hirt (1975) and Glimm et al. (1981) place the particles on the surface to be tracked. One of the most popular methods, the volume of fluid (VOF) (Hirt \& Nichols 1981), combines a convection equation for a scalar marker with a fixed grid.

Brackbill, Kothe \& Zemach (1992) developed the continuum surface force (CSF) method to include surface tension effects in the numerical treatment of problems with liquid/gas interfaces, adding a source term containing the surface tension action to the momentum differential equation. Alternatively, Lafaurie et al. (1994) and Zaleski (1996) considered this effect as a surface-tension tensor to be added to the viscous stress tensor. Both methods can be easily combined with finite volume methods for discretizing the conservation equations and with the VOF technique to follow the interface evolution.

The main objective of this paper is to uncover the mechanisms that trigger the sheet instability, and, particularly, to unveil the role of the interaction between the liquid-sheet oscillation and the gas-coflow dynamic structures and of vorticity. It is conjectured that the regular air vortex shedding process is probably related to the sheet motion and the latter will necessarily affect the former. We conduct a study of how the main parameters are likely to influence the development of the liquid-sheet instability.

To this end, this paper addresses the computation of the very-near field behind a nozzle tip injecting a thin, large-aspect-ratio liquid sheet into two symmetric highspeed gas coflows. The very-near field is defined as the region immediately behind the trailing edges of the nozzle plates where the vortex system originates. In this region, which typically extends downstream for around 5 to 10 times the nozzle width, the flow behaviour is dominated by the strong interaction between the vortical systems, and is markedly two-dimensional. The oversimplifications of existing linear and nonlinear studies for this region are overcome in this study by a general and complete formulation of the two-dimensional problem. The computational domain extends far upstream of the injection point; this allows the gas stream and liquid sheet perturbation to be triggered and grow in a natural way. As already mentioned in this Introduction, the flow visualizations obtained in the early 1990s clearly indicate a connection between the origin of the spanwise/streamwise liquid filaments and the existence of spanwise/streamwise vorticity structures behind the trailing edge of the splitter plates; these are strong indications that the gas-phase instabilities must have a direct bearing on the liquid-sheet deformation prior to breakup. In order to verify this, state-of-the-art numerical methods for single-phase flows are combined with wellestablished techniques for liquid/gas interface tracking and surface tension treatment. In order to compare the dynamics of the liquid-sheet/gas-coflow system with the gas-jet/gas-coflow one, a small part of the paper is dedicated to the computation of 

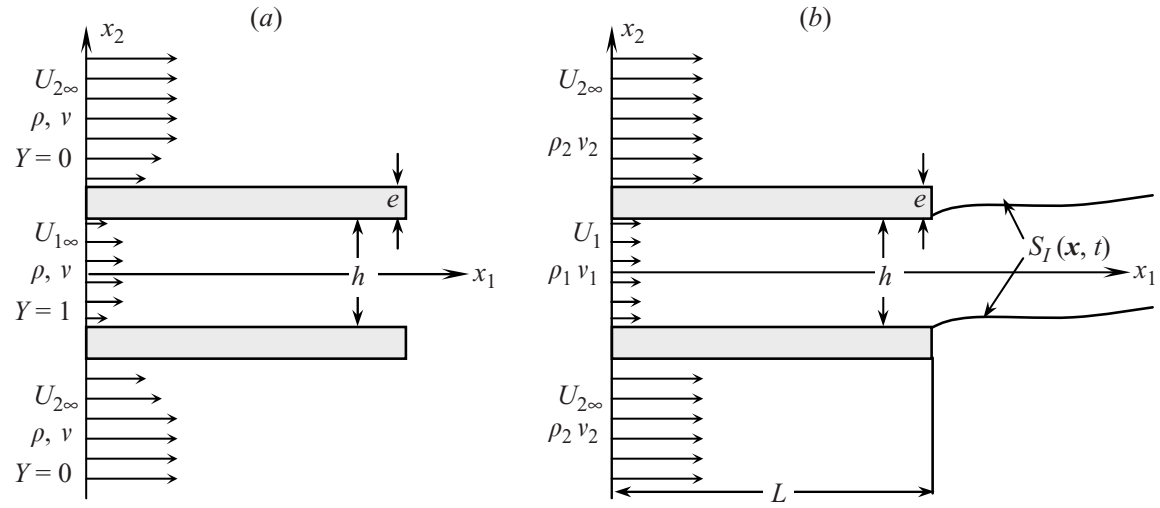

FIGURE 1. Two-dimensional single-phase gas-jet $(a)$ and two-phase liquid-sheet (b) geometries. The thickness $e$ may be zero for infinitely thin plates.

single-phase flows and its validation for the injection geometries investigated later for two-phase flows; simple experimental data are used for validation purposes. The calculations are two-dimensional and the main concern of this paper is with the injector very-near field, where the assumption of two-dimensionality might not be a gross oversimplification. Comparison of predictions and existing experimental data for sheet oscillation frequency and wavelength confirm that general trends are reproduced well with these two-dimensional computations.

Section 2 presents the mathematical formulation for both single-phase and twophase flows, including conservation equations, boundary and initial conditions; infinitely thin as well as thick solid plates are considered. The dimensionless singlephase and two-phase flow equations are obtained. The numerical techniques are presented in $\S 3$, with special attention on the use of accurate time discretization methods. The interface-tracking technique is next briefly described; the fractionalstep method adapted for this two-phase problem is also considered. The continuum surface force method used to numerically treat the surface tension as a part of the momentum equation is explained and the results of some validation tests are given.

Section 4 presents the numerical predictions for single-phase plane jets, first for thin-wall injectors and then for thick-walled nozzles. The results of the computations for thin liquid sheets sandwiched by two high-speed gas-streams are contained in $\S 5$; the influence of the gas Reynolds number, the Weber number and the plate thickness is extensively treated. Conclusions and future research are discussed in $\S 6$.

\section{Mathematical formulation}

2.1. Single-phase flow

\subsubsection{Governing equations}

A two-dimensional gas flow with constant density, $\rho$, and viscosity, $v$, between two plates leaves their trailing edges, merging with two coflows of the same gas constituting a gaseous jet (figure $1 a$ ). The plate thickness is $e$, and can be either finite or zero (infinitely thin plates). The separation between the plates is $h$. 
The dimensionless dynamic governing equations are

$$
\begin{aligned}
\nabla \cdot \boldsymbol{u} & =0, \\
S t \frac{\partial \boldsymbol{u}}{\partial t}+(\boldsymbol{u} \cdot \nabla) \boldsymbol{u} & =-\nabla p+\frac{1}{R e} \nabla \cdot \boldsymbol{\tau}^{\prime},
\end{aligned}
$$

where $\boldsymbol{u}=\left(u_{1}, u_{2}\right)$ represents the velocity vector, $p$ the pressure and $\boldsymbol{\tau}^{\prime}$ denotes the viscous stress tensor, expressed as

$$
\boldsymbol{\tau}^{\prime}=\left\{(\nabla \boldsymbol{u})+(\nabla \boldsymbol{u})^{\mathrm{T}}\right\} .
$$

In the above equations, $S t=h / U T$ and $R e=U h / v$ are the Strouhal and Reynolds numbers, respectively. Since the interest in this work is in the very-near field of the jet, its initial thickness, $h$, is taken as the characteristic length and $U$ (either $U_{1 \infty}$ or $\left.U_{2 \infty}\right)$ as the characteristic velocity. $T$ is a characteristic period of vortex shedding or jet oscillation, for example.

A species with mass fraction $Y=1$ between the plates and $Y=0$ in the coflows is considered in order to follow the mixing patterns; $Y$ is sometimes termed the mixture fraction, and obeys the transport equation:

$$
S t \frac{\partial Y}{\partial t}+(\boldsymbol{u} \cdot \nabla) Y=\frac{1}{S c R e} \nabla^{2} Y,
$$

where $S c=v / D$ is the scalar Schmidt number, and $D$ the diffusion coefficient.

\subsubsection{Boundary and initial conditions}

On the solid surfaces the no-slip condition $\boldsymbol{u}=0$ is imposed. At $x_{1}=0$ two Blasius profiles are assumed for the coflows; this is equivalent to starting the computation upstream $\left(x_{1}<0\right)$ and allowing the development of two boundary-layer-type profiles. The resulting momentum thickness will be used, in a Reynolds number, to characterize the case. Between the plates, $-0.5 h<x_{2}<0.5 h$, flows with either fully developed velocity profiles or two developing boundary layers are considered. Far away from the plates $u_{1} \rightarrow U_{2 \infty}$.

Transient computations are performed starting from trivial initial fields $u_{1}(\boldsymbol{x}, 0)=0$, $u_{2}(\boldsymbol{x}, 0)=0, p(\boldsymbol{x}, 0)=0$ and $Y(\boldsymbol{x}, 0)=0$.

\subsection{Two-phase flow}

\subsubsection{Governing equations}

A two-dimensional thin liquid sheet flows between two plates and is injected at their trailing edges into two high-speed gas coflows as shown in figure $1(b)$. The thickness of the liquid sheet is $h$, and the plates have a thickness $e$, which can be finite or zero (infinitely thin plates). The liquid and the gas coflows enter the domain at $x_{1}=0$ with uniform velocities $U_{1 \infty}$ and $U_{2 \infty}$, respectively.

The velocity vector, the pressure, the density and the kinematic viscosity of phase $\alpha$ are designated $\boldsymbol{u}_{\alpha}=\left(u_{1 \alpha}, u_{2 \alpha}\right), p_{\alpha}, \rho_{\alpha}$ and $v_{\alpha}$ respectively, where $\alpha=1$ for the liquid phase and $\alpha=2$ for the gas coflows. Considering both phases incompressible, the dimensionless governing equations are

$$
\begin{aligned}
\nabla \cdot \boldsymbol{u}_{\alpha} & =0 \\
S t_{\alpha} \frac{\partial \boldsymbol{u}_{\alpha}}{\partial t}+\left(\boldsymbol{u}_{\alpha} \cdot \nabla\right) \boldsymbol{u}_{\alpha} & =-\nabla p_{\alpha}+\frac{1}{R e_{\alpha}} \nabla \cdot \boldsymbol{\tau}_{\alpha}^{\prime},
\end{aligned}
$$

where

$$
\boldsymbol{\tau}_{\alpha}^{\prime}=\left\{\left(\nabla \boldsymbol{u}_{\alpha}\right)+\left(\nabla \boldsymbol{u}_{\alpha}\right)^{\mathrm{T}}\right\}
$$


is the viscous stress tensor and $S t_{\alpha}=\omega_{\alpha} l_{\alpha} / U_{\alpha}$, and $R e_{\alpha}=U_{\alpha} l_{\alpha} / v_{\alpha}$ are, respectively, the Strouhal and the Reynolds numbers for phase $\alpha ; \omega_{1}$ is the liquid-sheet oscillating frequency, while $\omega_{2}$ is the vortex shedding frequency; $l_{1}$ is either the unperturbed sheet thickness, $h$, for fully developed viscous flow or the liquid boundary-layer momentum thickness, $\theta_{1}$, for entrance-type flows; $l_{2}$ may be either the gaseous boundary-layer momentum thickness, $\theta_{2}$, or the plate thickness, $e \cdot U_{2}$ is $U_{2 \infty}$ for the gas coflows, and $U_{1}$ is $U_{1 \infty}$.

\subsubsection{Boundary and initial conditions}

At the entrance to the fluid domain, $x_{1}=0$, uniform velocity profiles are assumed:

$$
\begin{aligned}
& \boldsymbol{u}_{11}=1 \quad \text { for } \quad-\frac{1}{2} h \leqslant x_{2} \leqslant \frac{1}{2} h, \\
& \boldsymbol{u}_{12}=1 \quad \text { for } \quad x_{2}<-\left(\frac{1}{2} h+e\right) \text { and } x_{2}>\left(\frac{1}{2} h+e\right) .
\end{aligned}
$$

The velocity profiles then evolve along the plate from $x_{1}=0$ to $x_{1}=L$, with developing boundary layers for $\alpha=1$ and $\alpha=2$. Far away from the plates, within the gas coflows, $\boldsymbol{u}_{12} \rightarrow 1$ for $x_{2} \rightarrow \pm \infty$. On the solid plate surfaces $\boldsymbol{u}_{\alpha}=0$ for $\alpha=1$ and 2 .

At the interface, the kinematic boundary conditions are

$$
\boldsymbol{u}_{1}=\frac{U_{2 \infty}}{U_{1 \infty}} \boldsymbol{u}_{2}
$$

and

$$
S t_{\alpha} \frac{\partial S_{I}}{\partial t}+\boldsymbol{u}_{\alpha} \cdot \nabla S_{I}=0
$$

The dimensionless dynamic stress balance condition is

$$
\left(p_{1}-M p_{2}\right) \boldsymbol{n}=\left(\frac{1}{R e_{1}} \boldsymbol{\tau}_{1}^{\prime}-\frac{M}{R e_{2}} \boldsymbol{\tau}_{2}^{\prime}\right) \cdot \boldsymbol{n}-\frac{\kappa}{W e} \boldsymbol{n}
$$

where the pressures at $\alpha=1$ and $\alpha=2$ are made dimensionless by $\rho_{1} U_{1 \infty}^{2}$ and $\rho_{2} U_{2 \infty}^{2}$, respectively, $M$ is the momentum flux ratio $M=\rho_{2} U_{2 \infty}^{2} / \rho_{1} U_{1 \infty}^{2}, \kappa=-\nabla \cdot \boldsymbol{n}$ is the mean surface curvature made dimensionless with either $h$ or $\theta_{1}$ and $W e=\rho_{1} U_{1 \infty}^{2} \theta_{1} / \sigma$ is the Weber number, $\sigma$ being the constant surface tension coefficient.

The initial conditions for the two-phase calculations are as follows. At $t=0$, a flat (unperturbed) liquid sheet occupies the region $-h / 2 \leqslant x_{2} \leqslant h / 2$; the rest of the domain is occupied by air. The liquid sheet is moving with the same velocity profile as the inlet one (i.e. a top-hat profile) while the surrounding gas is considered at rest. From these unperturbed initial fields, the perturbations in the flow develop in a natural way after some transient time.

\section{Numerical technique}

\subsection{Single-phase flow numerical techniques}

A finite-volume method is employed to discretize the governing equations and the corresponding boundary and initial conditions. A structured, Cartesian, staggered mesh is used. All the model transport equations are considered to be of the following common form, which is expressed in dimensional form so that the nomenclature commonly used in the field of computational fluid dynamics can be retained:

$$
\frac{\partial \rho \varphi}{\partial t}=-\nabla \cdot\left(\rho \boldsymbol{u} \varphi-D_{\varphi} \nabla \varphi\right)+S_{\varphi}=H(\varphi, t)
$$


where $D_{\varphi}$ is the molecular-diffusion coefficient for variable $\varphi$ and $S_{\varphi}$ stands for all the source terms.

Centred differences are used to discretize the diffusive flux of $\varphi$ and the convective flux through a given cell face is calculated by using a combination of a first-orderaccurate, unconditionally stable term (the upwind scheme, Patankar 1980) and a second-order anti-diffusive correction term; for the latter, a variation of the SMART scheme (Gaskell \& Lau 1988), employing a flux limiting function (Waterson \& Deconnick 1994), is adopted.

\subsubsection{Time discretization}

The conservation equation for $\varphi$ has been discretized using several temporal schemes. In general, a three-time-level approach is used, given by the equation

$$
\left(1+q_{1}\right) \varphi^{(n)}-\left(1+2 q_{1}\right) \varphi^{(n-1)}+q_{1} \varphi^{(n-2)}=\Delta t\left[q_{2} H^{(n)}+\left(1-q_{2}-q_{3}\right) H^{(n-1)}+q_{3} H^{(n-2)}\right],
$$

where $\Delta t$ is the value of the $n$th time step, $\varphi^{(n)}$ and $H^{(n)}$ are the values of $\varphi$ and of the right-hand side of equation (11) evaluated at time $t^{(n)}$, and $q_{1}, q_{2}$ and $q_{3}$ are numerical parameters. For $q_{3}=q_{1}-q_{2}+1 / 2$, the scheme is second-order accurate; moreover, if in addition $q_{1}=2 q_{2}-5 / 6$ the scheme is a third-order accurate (Hirsch 1988).

The Euler explicit and implicit schemes are obtained if $q_{1}=q_{2}=q_{3}=0$ and $q_{1}=q_{3}=0, q_{2}=1$, respectively. If $q_{1}=q_{3}=0, q_{2}=1 / 2$ the Crank-Nicholson scheme is recovered. The third-order scheme results from the assignment $q_{1}=-1 / 6, q_{2}=-1 / 3$ and $q_{3}=0$. López-Pagés (2000) has assessed the performance of all four schemes for a gaseous mixing layer behind a splitter plate; while the first-order schemes do not reproduce some of the flow features encountered in previous experimental investigations (Meiburg \& Lasheras 1988), the second- and third-order ones correctly capture the structure of the two-dimensional vortices very near the trailing edge of the splitter plate; however, the fluctuating frequencies calculated with the three schemes at three different downstream locations do not differ significantly.

\subsubsection{Other solution details}

The discretized equations are solved using a segregated solver. The SIMPLEST algorithm (a variant of the SIMPLE algorithm, Patankar 1980) is used to calculate pressure from the momentum and continuity equations. SIMPLEST differs from other SIMPLE-like algorithms in that the diffusive and convective contributions to the $A_{k}$ coefficients in the momentum equations are treated separately: while the diffusive part is treated implicitly, the convective contribution is treated explicitly; thus, the finite-volume coefficients become reciprocal, and the convergence rate is accelerated.

The linear-equation solver is a two-dimensional, iterative-version of the Thomas or TDMA algorithm which is similar in concept to the SIP procedure (Stone 1968). Relaxation (either implicit or explicit) is used for all the variables to promote convergence.

\subsection{Numerical techniques for two-phase flows}

Two features, namely the tracking of the interfaces and the treatment of the surface tension, are specific to the two-phase flows considered in this research and therefore special consideration is given to them in this section. Dimensional equations are used in this section for the reasons indicated above for single-phase flows.

\subsubsection{Interface tracking}

The volume of fluid (VOF) method is used for tracking the interface. A scalar field $\Phi(\boldsymbol{x}, t)$ is defined, such that, for example, $\Phi=1$ for the liquid phase and $\Phi=0$ for the 
gas phase. For a known $\Phi$ field, the interface is determined by intermediate values of the scalar field, $0<\Phi(\boldsymbol{x}, t)<1$. The scalar $\Phi$ obeys the conservation equation:

$$
\frac{\partial \Phi}{\partial t}+\nabla \cdot u \Phi=0
$$

For numerical purposes, a single auxiliary fluid may be defined in terms of $\Phi$ with density and viscosity respectively equal to

$$
\rho_{\Phi}=\Phi \rho_{1}+(1-\Phi) \rho_{2}, \quad \mu_{\Phi}=\Phi \mu_{1}+(1-\Phi) \mu_{2}
$$

The velocity field, $\boldsymbol{u}$, to be used in equation (13) is provided by the governing equations

$$
\begin{gathered}
\nabla \cdot \boldsymbol{u}=0, \\
\rho_{\Phi} \frac{\partial \boldsymbol{u}}{\partial t}+\rho_{\Phi}(\boldsymbol{u} \cdot \nabla) \boldsymbol{u}=-\nabla p+\nabla \cdot \boldsymbol{\tau}^{\prime}+\sigma \kappa \boldsymbol{n} \delta\left(S_{I}\right),
\end{gathered}
$$

where $\boldsymbol{\tau}^{\prime}=\mu_{\Phi}\left\{(\nabla \boldsymbol{u})+(\nabla \boldsymbol{u})^{T}\right\}$ and the surface tension is included in the momentum equation (16) as a force concentrated at the interface $S_{I}$ by means of a Dirac delta function $\delta\left(S_{I}\right)$.

For the spatial discretization, first-order upwind differencing and second-order van Leer (1979) schemes are customarily used. For flows with liquid/gas interfaces, Hirt \& Nichols (1981) propose a scheme (HN hereafter) based on the donor-acceptor scheme of Ramshaw \& Trapp (1976). In order to determine the interface orientation within a finite-volume cell, this is approximated by a straight-line segment in two-dimensional flows, or by a plane portion in three-dimensional ones. The normal vector to the interface is calculated in terms of $\Phi$ as: $\boldsymbol{n}=\nabla \Phi$.

López-Pagés (2000) has shown that the HN scheme is more accurate than the second-order van Leer one, and that it retains its accuracy with non-uniform Cartesian grids; further, it is easily generalized to three-dimensional codes.

\subsubsection{Time integration}

Apart from the limitations on the time step size imposed by the explicit treatment of equation (13), its time integration scheme may be a source of errors in tracking the interface. The fractional-step technique (Yanenko 1971; Hirsch 1988; Barley 1988) is used to alleviate this difficulty. Equation (13) may be discretized in two dimensions as

$$
\Phi^{(n)}=L_{1}^{(n)} L_{2}^{(n)} \Phi^{(n-1)}+O\left(\Delta t^{(n)^{2}}\right)
$$

where $L_{i}^{(n)}=1+\Delta t^{(n)} S_{i}^{(n)}, S_{i}^{(n)}=-u_{i}(\boldsymbol{x}, t) \partial / \partial x_{i}$ and $i=1,2$.

Several combinations of the operators $L_{1}^{(n)}$ and $L_{2}^{(n)}$ are possible in order to reproduce the action of the spatial operators $S_{1}^{(n)}$ and $S_{2}^{(n)}$. In this work a version of the Yanenko sequential scheme is used, namely

$$
\Phi^{(n)}=L_{1}^{(n / 2)} L_{2}^{(n / 2)} L_{2}^{(n / 2)} L_{1}^{(n / 2)} \Phi^{(n-1)},
$$

where $L_{1}^{(n / 2)}=1+\left(\Delta t^{(n)} / 2\right) S_{1}^{(n)}$ and $L_{2}^{(n / 2)}=1+\left(\Delta t^{(n)} / 2\right) S_{2}^{(n)}$.

\subsubsection{Numerical treatment of surface tension}

The continuum surface force (CSF) method (Brackbill et al. 1992) is used in this work in combination with the finite-volume discretization of the conservation equations and the VOF technique for interface tracking. The last term in equation (16) represents the surface tension acting on every control volume containing a portion of 


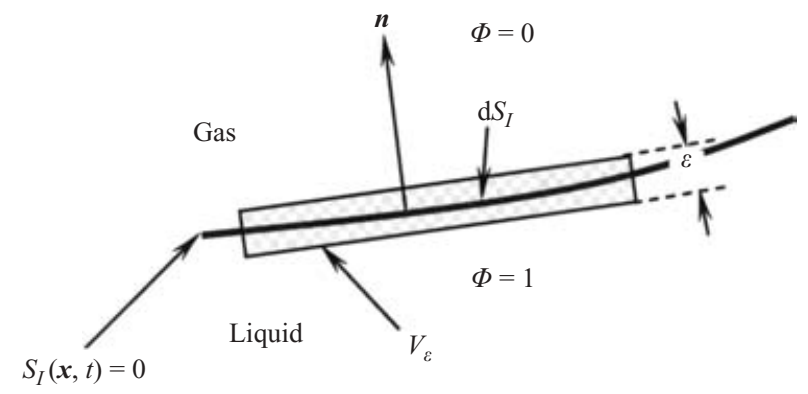

FIGURE 2. Integration volume $V_{\varepsilon}$ for an interface element $\mathrm{d} S_{I}$.

the interface (see figure 2); in the CSF method that term is determined by the relation $-\nabla \tilde{\Phi}=\boldsymbol{n} \delta\left(S_{I}\right)$. The function $\tilde{\Phi}(\boldsymbol{x}, t)$ is known as the smoothed scalar field and it is obtained as an average of $\Phi$ in neighbouring cells ( $W, E, S$, and $N$ )

$$
\tilde{\Phi}_{P}=C_{1} \Phi_{P}+C_{2}\left(\Phi_{W}+\Phi_{E}+\Phi_{S}+\Phi_{N}\right)+C_{3}\left(\Phi_{N E}+\Phi_{N W}+\Phi_{S E}+\Phi_{S W}\right),
$$

where $C_{1}=0.5, C_{2}=0.35$ and $C_{3}=0.15$.

For the surface-tension term, the normal to the interface is obtained in terms of $\tilde{\Phi}$. A normal vector is assigned to every corner of the cell containing the interface; the normal components at any mesh node are obtained by averaging the values at the four corners. Next, the mean interface curvature, $\kappa=-\nabla \cdot \boldsymbol{n}$ is obtained.

When surface tension is included in the calculation, the time step used must allow resolution of the propagation of the capillary mode with the largest wavenumber vector, namely

$$
k_{1 m}=\frac{\pi}{\Delta x_{1 \min }}, \quad k_{2 m}=\frac{\pi}{\Delta x_{2 \min }},
$$

where $\Delta x_{1 \min }=\min \left(\Delta x_{1 P}\right)$ and $\Delta x_{2 \min }=\min \left(\Delta x_{2 P}\right)$, for all grid points. If the speed of an interfacial capillary wave is expressed as $c_{1}=\sigma \kappa / \rho_{1}$, the time step size to resolve the capillary mode is given by

$$
\Delta t_{P}=\min \left\{\left(\frac{\rho_{1}\left(\Delta x_{1 P}\right)^{3}}{2 \pi \sigma}\right)^{1 / 2},\left(\frac{\bar{\rho}_{1}\left(\Delta x_{2 P}\right)^{3}}{2 \pi \sigma}\right)^{1 / 2}\right\},
$$

with

$$
\Delta t_{S T} \leqslant \min \left(\Delta t_{P}\right)
$$

for all grid points. Details on the numerical implementation of this procedure can be found in López-Pagés (2000).

Figure 3 shows the evolution of an initially almost square liquid domain being convected along its diagonal, $\left(u_{1}, u_{2}\right)=(1,1)$, under the action of the surface tension. The fractional-step method is used for the time integration, while the HN scheme is employed for the convective fluxes. The large value of the surface force at the corners causes the liquid to adopt a shape where the corners are located where the sides had previously been. For large times, a circular two-dimensional drop results under the action of the viscosity and the surface tension. A generalization to three dimensions is shown by López-Pagés (2000). 

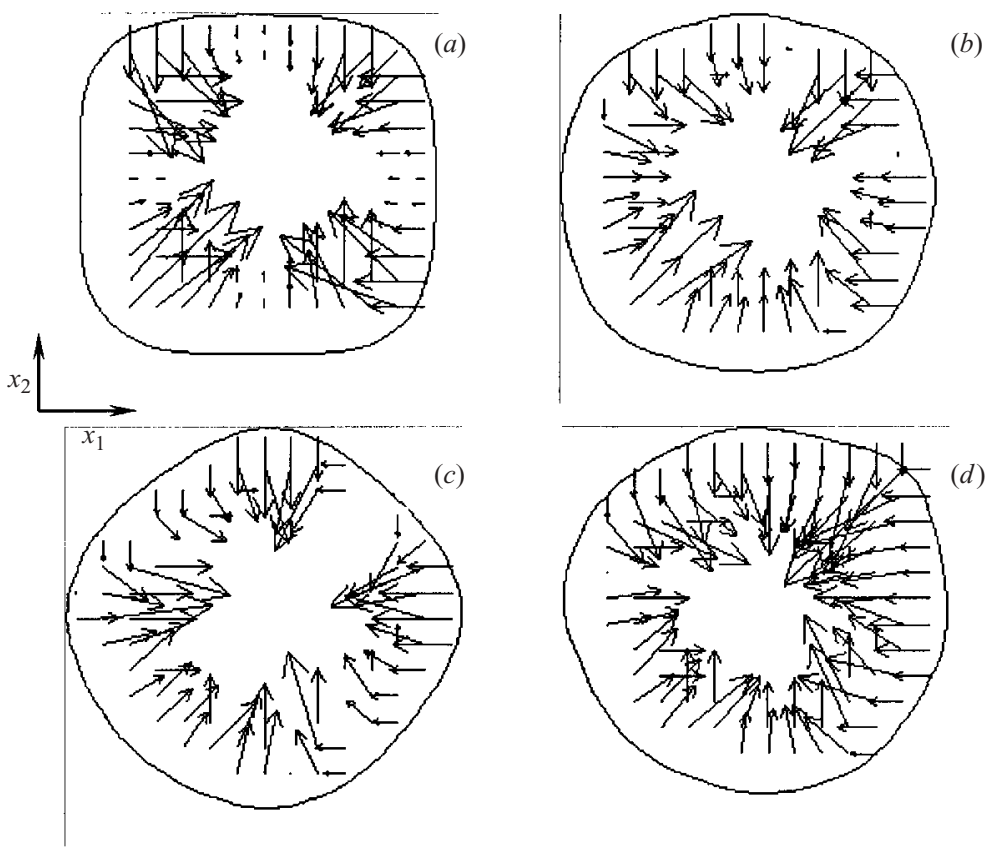

FIGURE 3. Restoring action of the surface-tension force: contours of smoothed scalar marker field $\tilde{\Phi}$ with superimposed surface-tension-force vectors at four times: $(a) t=t_{0},(b) t=2 t_{0}$, (c) $t=3 t_{0}$ and (d) $t=4 t_{0}$.

\subsection{Computational domain and boundary conditions}

All the calculations reported in this paper focus on the near-field region of the nozzle tip. In order to minimize any unwanted numerical influence of the boundary conditions on the phenomena taking place in the near-field region, the computational domain is chosen to be much larger than the liquid sheet thickness $h$ (typically 30 times in the longitudinal direction and 20 in the transverse one).

Boundary conditions are implemented implicitly. At the domain inlets, mass flow rates and momentum fluxes are prescribed using the profiles detailed in $\S \S 2.1 .2$ and 2.2.2 above. At the other domain boundaries (downstream and top and bottom planes), pressure-type boundary conditions are used. The pressure just outside the domain is presumed to be the reference one (zero for this incompressible flow), and an outflow/inflow results when the in-cell pressure is respectively above/below the outside pressure. Since the outflow at the exit boundaries is not fully developed, and indeed there can even be an inflow, a zero-gradient boundary condition would provide unphysical results.

\section{Results for single-phase jets}

López-Pagés (2000) has validated the finite-volume code for single-phase flows, and has used it extensively to analyse mixing layers behind thin and thick trailing edges, as well as two-dimensional jets with thin and thick wall injectors. Pressure profiles along the flow direction reproduce the shapes obtained by asymptotic expansion techniques (Stewartson 1969; Smith 1982) for two-dimensional wakes or mixing layers behind a thin plate; the cross-stream profiles of the longitudinal flow component and the mixture fraction correctly collapse on a single non-dimensional curve. Using a 

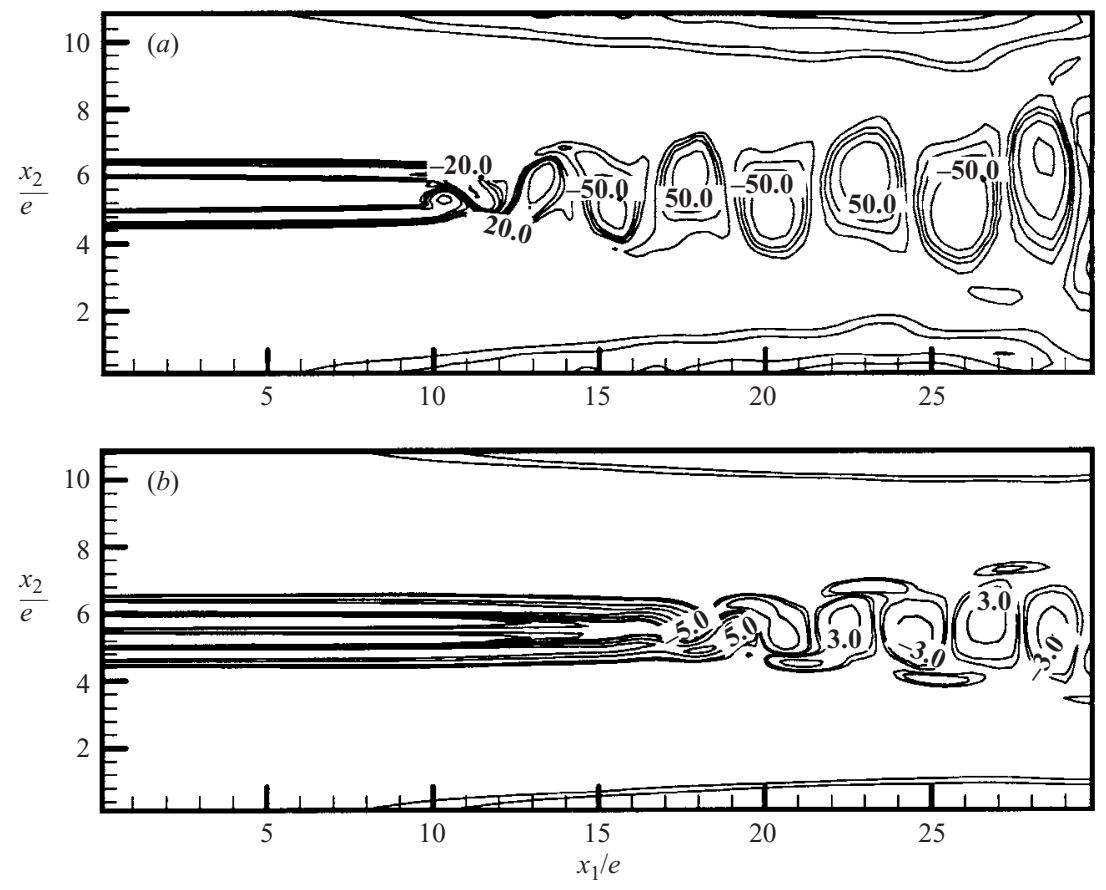

FIGURE 4. Single-phase case: vorticity contours for two velocity ratios: $U_{2 \infty} / U_{1 \infty}$ : (a) 30 and (b) 3 . Vorticity values are made dimensionless with $e U_{100}^{-1}$.

third-order implicit time-discretization scheme, the vortex structure visualized by Meiburg \& Lasheras (1988) in the mixing layer of a thick plate between two streams with equal velocities is correctly captured by the code; the trends of the Strouhal number as a function of the Reynolds number are also predicted well (Oertel 1990). An extensive study of mixing layers behind thin- and thick-walled plates, varying the Reynolds numbers, the velocity ratios and the density ratios of the two streams, has been systematically conducted.

The main results for two-dimensional single-phase jets are presented now. The aim of this part of the study is to investigate the role of the plate thickness in the onset and sustainability of the instability, and to serve as a basis for the later comparison with the liquid-sheet/gas-coflow system.

We turn our attention first to the case where a gas jet is sandwiched between two equal infinite gas coflows with infinitely thin solid plates (figure $1 a, e=0$ ). When the three streams have identical velocities, López-Pagés (2000) has shown that the merging of the three streams at the plate trailing edges causes the appearance of instabilities in the neighbourhood of the injection and downstream of it; however the flow conditions do not allow for the maintenance of the instability and the perturbations eventually die for all Reynolds numbers investigated $\left(R e_{L}=650,3240\right.$, 6480, 12960 and 19430). López-Pagés (2000) has noted that the perturbation survival time increases with the Reynolds number.

Unequal velocities in the merging streams allow the instability to be sustained. Figure 4 shows the non-dimensional vorticity patterns for the two velocity ratios $U_{2 \infty} / U_{1 \infty}=30$ and 3 , and both cases are unstable. The more detailed study by López-Pagés (2000) shows the flow to be unstable for $U_{200} / U_{100} \geqslant 3$. It also shows, as illustrated by figure 4 , that vortex formation starts further downstream as the velocity 
(a)
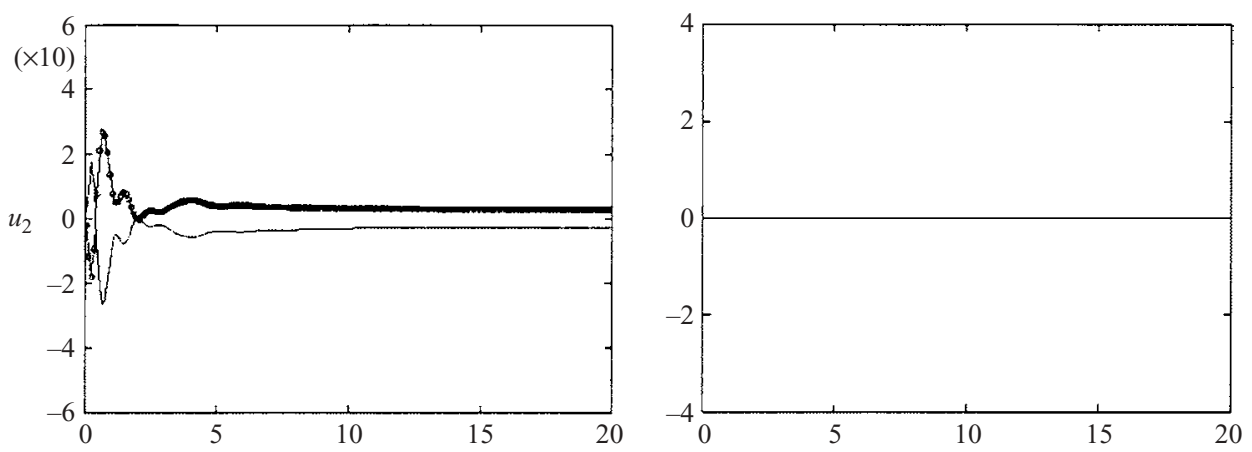

(b)
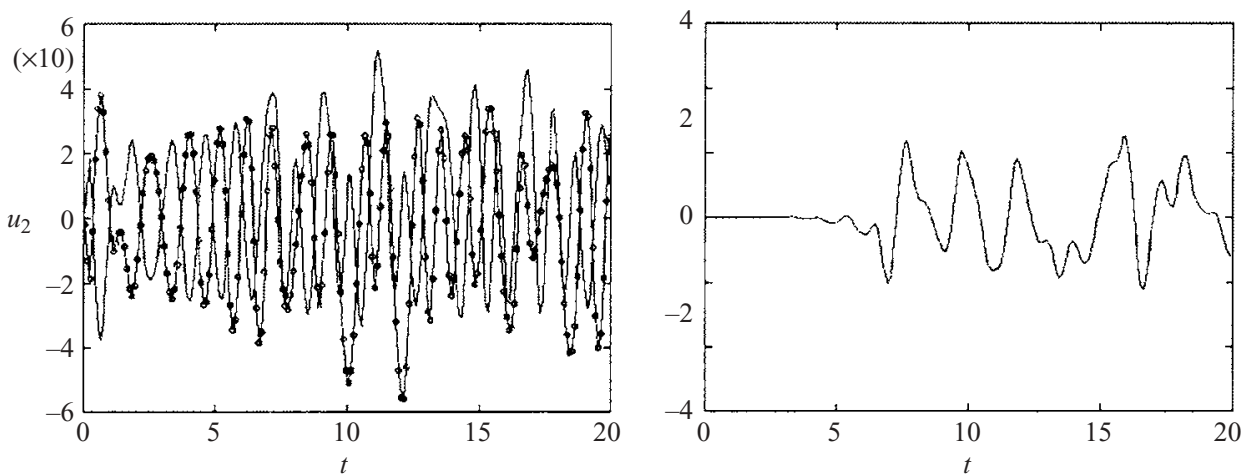

FIGURE 5. Single-phase case: transient evolution of the transverse velocity component, $u_{2}$, at $x_{1}=L$ and $x_{2}= \pm 0.5(h+e)$ (left) and $x_{2}=0$ (right) for $(a) R e_{L}=650$, and (b) 19 430. Coarser dots in the left-hand images represent values at the negative $x_{2}$ coordinate.

ratio decreases. For the larger velocity ratio, vortex formation takes place immediately after the trailing edges, due to the momentum deficit in the central stream.

We now turn our attention to the case where the streams are separated by thick plates (figure $1 a$, with $e / h=1$ ). Figure 5 shows the transient time evolution of the transverse velocity component, $u_{2}$, at the jet exit for a velocity ratio $U_{2 \infty} / U_{1 \infty}=1$ and two different Reynolds numbers, $R e_{L}=650$ and 19430. (The case $R e_{L}=3240$ was also studied, but the results are not shown.) In contrast with the zero-thickness case, which is stable for all $R e_{L}$ when the velocity ratio is $U_{2 \infty} / U_{1 \infty}=1$, this finite-thickness case shows the development of sustained instabilities for all except for the smallest $R e_{L}$, which yields damped perturbations. The time for the initiation of the velocity perturbation at the jet centreline decreases as the Reynolds number increases. The time evolutions at the two points $x_{2}= \pm 0.5(h+e)$ are half a period out of phase, until the perturbation growth starts. As a consequence of the jet/wake structure of this flow, two characteristic frequencies are measurable, namely $f_{0}=0.4$ and 1.4 (at the jet centreline) and $f_{ \pm}=0.4$ and 2.8 (at the other two points) for $R e_{L}=3240$ and 19430 , respectively. Figure 6 displays the corresponding vorticity isocontours. The interaction between the jet and the coflows also presents some features of wake structures, and the mixed jet/wake structure of this flow is apparent from the vorticity isocontours. For $R e_{L}$ smaller than 12960 (among those investigated by López-Pagés 2000), there is only a single flow frequency; for $R e_{L}=19430$ two frequencies exist. The interaction 

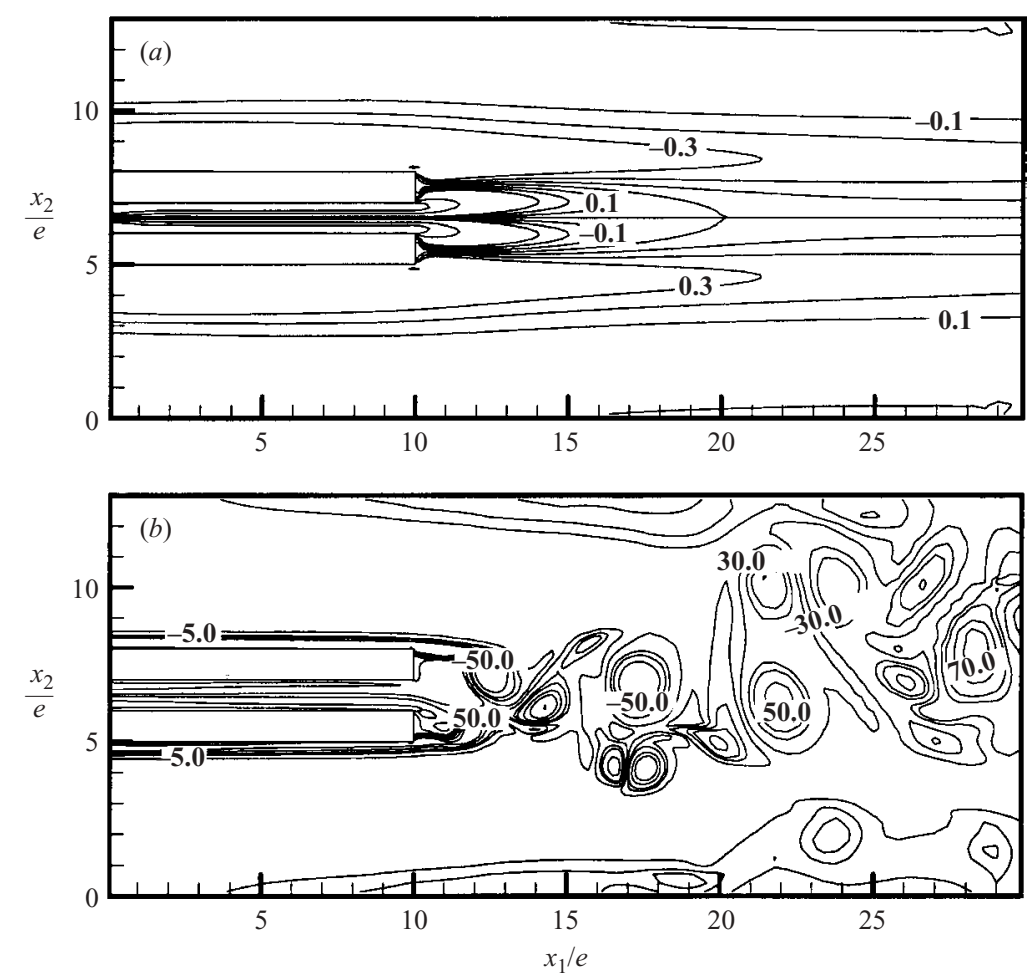

FIGURE 6. Single-phase case: vorticity contours for two Reynolds numbers: (a) 650 and (b) 19430. Vorticity values are made dimensionless with $e U_{1 \infty \infty}^{-1}$.

among the vortical systems shed from the four corners at the trailing edges gives rise to the presence of the subharmonic. Figure 6 shows the difference between an ordered flow with well-defined attached recirculation zones for $R e_{L}=650$ and complicated vorticity patterns for $R e_{L}=19430$. The Strouhal number as a function of the Reynolds number is plotted in figure 7. For $R e_{e}$ (Reynolds number based on plate thickness) smaller than approximately 1300 the curve is monotonically increasing, as customary in wake flows. The decrease for $R_{e}$ greater than 1300 is probably associated with the double jet/wake nature of the flow structure.

The role played by the velocity ratio is examined next. Figure 8 presents the transient evolution of the transverse velocity for three velocity ratios, $U_{2 \infty} / U_{1 \infty}=30,3$ and 1 and the corresponding vorticity isocontours. For $U_{2 \infty} / U_{1 \infty}=30$, the jet velocity is so small compared to that of the coflows that the jet/coflow interaction is rather limited. From the animation of the computational results, it is evident that the jet hardly penetrates the flow downstream of the trailing edges; the intense vortex shedding from the upper and lower corners immediately disrupts the jet entering the flow at $x_{1}>L$. The vorticity contours are qualitatively similar to those obtained for the wake flow behind a single obstacle of thickness $(h+2 e)$.

As the velocity ratio decreases $\left(U_{2 \infty} / U_{1 \infty}=3\right)$ a small jet/coflow interaction is apparent; the transient perturbations of transverse velocity at the jet centreline start earlier. Further reductions of the velocity ratio increase the interactions among the three streams after they merge, decreasing both the time of appearance of velocity fluctuations and their amplitude; smaller vorticity structures control the flow dynamics and a loss of spatial periodicity is observed. Unlike the same flow with infinitely 


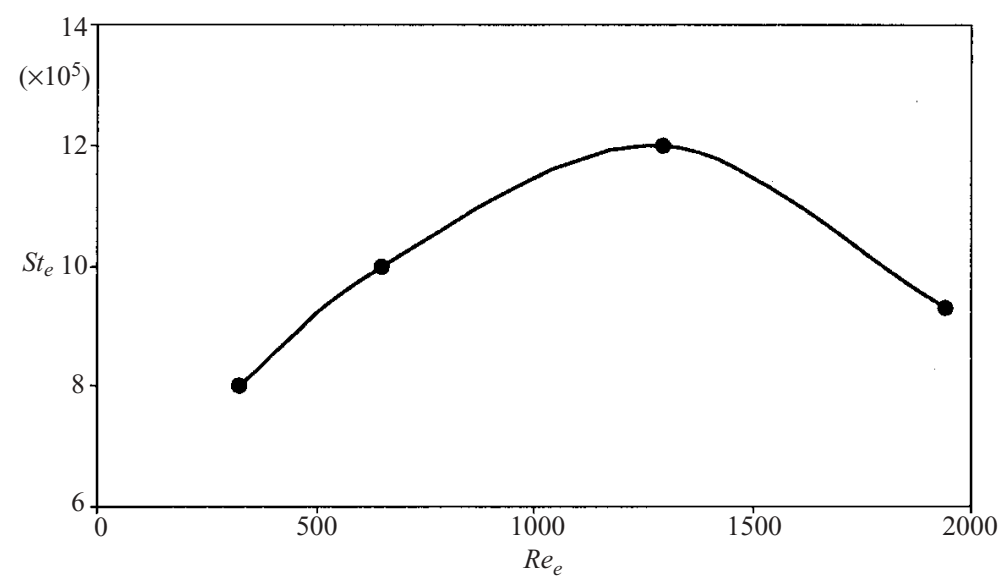

FIGURE 7. Strouhal number, $S t_{e}=e f / U_{2 \infty}$, as a function of the Reynolds number, $R e_{e}=e U_{2 \infty} / v$, for the gas-jet/wake flows.

$\begin{array}{cccc}U_{2 \infty} / U_{1 \infty} & f_{+}(\mathrm{kHz}) & f_{-}(\mathrm{kHz}) & f_{0}(\mathrm{kHz}) \\ 30 & 1.5-3.0 & 1.5-3.1 & 1.5 \\ 6 & 1.7-3.4 & 1.7-3.3 & 1.7 \\ 3 & 3.3 & 3.5 & 1.7 \\ 1.5 & 2.6 & 2.7 & 1.5 \\ 1.0 & 2.8 & 2.8 & 1.4\end{array}$

TABLE 1. Characteristic frequencies for the transverse velocity oscillation behind the injection plates for five velocity ratios; $f_{+}$for upper plate; $f_{-}$for lower plate; $f_{0}$ at the jet centreline (single-phase flow).

thin plates, the flow is now unstable for a velocity ratio of 1 (figure $8 c$ ). For the characteristic frequencies, the results in table 1 are obtained; no saturation frequency is reached. For the two larger velocity ratios, subharmonics are present in the velocity perturbations just behind the plates; these frequencies also identify the fluctuations at the jet centreline, with values very close to those corresponding to the vortex shedding behind a thick plate (López-Pagés 2000). As the velocity ratio decreases no subharmonics are detected just behind the plates, and $f_{0}$ at the jet centreline is now the only subharmonic.

\section{Results for liquid sheets}

In this Section the results for the dynamics of a thin liquid sheet (a liquid jet) sandwiched between two high-speed gas coflows are presented. The properties (density and viscosity) of water and air at ambient temperature and pressure are taken for the liquid and gas, respectively. For the velocities in this study, the gas is considered incompressible.

Only two-dimensional cases are studied, which may affect the comparison of the numerical predictions with three-dimensional experimental results; for instance, the experimentally observed streamwise liquid filaments cannot be predicted. However, this study investigates the very-near field behind the injection point, where the 


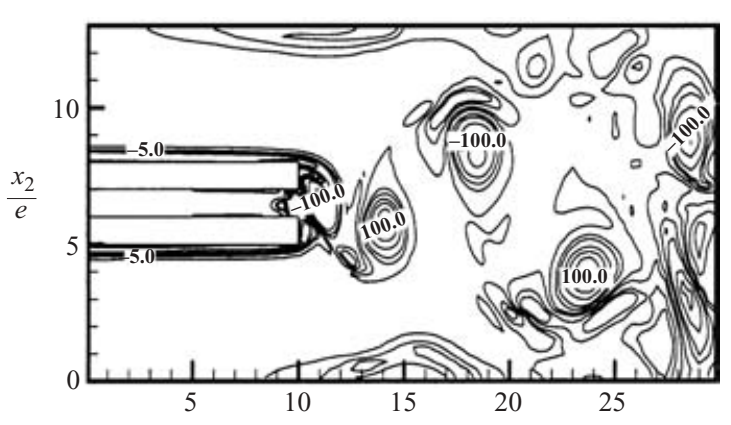

(a)
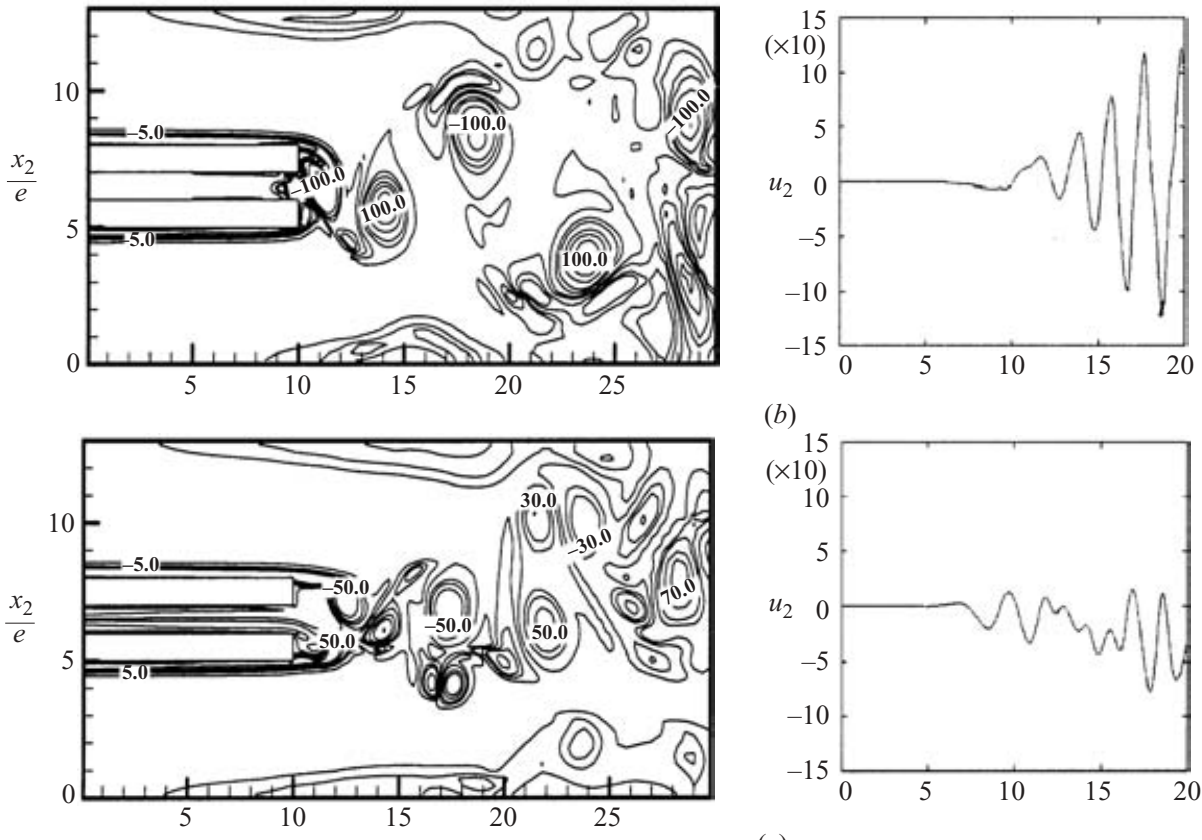

(b)

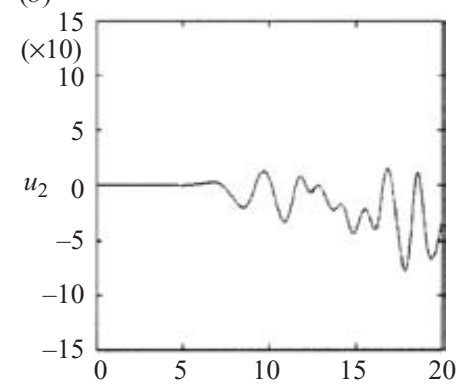

(c)
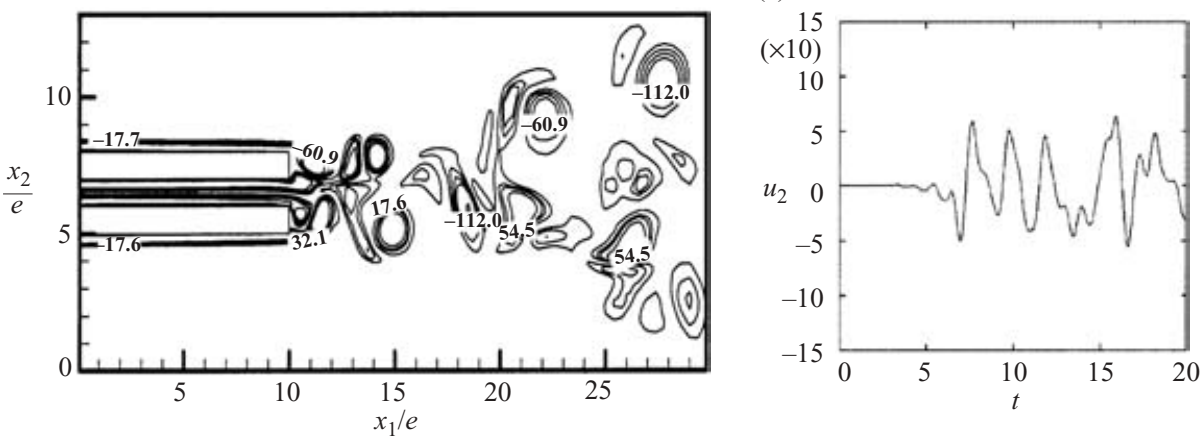

FIGURE 8 . Single-phase case: vorticity contours and transient time evolution of the transverse velocity at $x_{1}=L$ (left) and $x_{2}=0$ (right) for velocity ratios $U_{2 \infty} / U_{1 \infty}:(a) 30,(b) 3$ and (c) 1 . Vorticity values are made dimensionless with $e U_{1 \infty}^{-1}$.

characteristic flow structures may well be considered approximately two-dimensional. This work is expected to be a highly relevant first step to unveil the way in which the liquid sheet instability is triggered as the liquid and gas streams merge.

In this section, the boundary-layer momentum thickness for the liquid, $\theta_{1}$, and its counterpart for the gas coflows, $\theta_{2}$, are often used as the reference length scale. These are numerically calculated at the exit of the plates, from the boundary-layer profiles which develop on the liquid and fluid sides of the plate.

This section investigates the role in the instability of the main flow parameters, i.e. the plate thickness, the coflow Reynolds number, the surface tension, and the slip condition for the contact line. Table 2 summarizes the values of the flow parameters in the cases studied. The location of the liquid sheet in the pictures is determined by using scalar marker, $\Phi$, isocontours to trace the interface location. 


\begin{tabular}{cccccccc}
\hline Case & Thickness & $U_{2 \infty}\left(\mathrm{m} \mathrm{s}^{-1}\right)$ & \multicolumn{1}{c}{$M$} & $\theta_{2}(\mathrm{~m})$ & $\operatorname{Re}_{\theta_{1}}$ & $R e_{\theta_{2}}$ & $W e$ \\
1 & Thick & 10 & 0.12 & $8.25 \times 10^{-5}$ & 66 & 53 & - \\
2 & Thick & 20 & 0.48 & $5.83 \times 10^{-5}$ & 66 & 76 & - \\
3 & Thick & 30 & 1.07 & $4.76 \times 10^{-5}$ & 66 & 92 & - \\
4 & Thick & 50 & 2.97 & $3.67 \times 10^{-5}$ & 66 & 119 & - \\
5 & Thick & 100 & 11.89 & $2.61 \times 10^{-5}$ & 66 & 169 & - \\
6 & Thick & 30 & 1.07 & $4.76 \times 10^{-5}$ & 66 & 92 & 0.9 \\
7 & Thin & 30 & 1.07 & $4.76 \times 10^{-5}$ & 66 & 92 & -
\end{tabular}

TABLE 2. Characteristic values of the parameters used for each computational simulation for the liquid-sheet/gas-coflow system. $U_{1 \infty}=1 \mathrm{~m} \mathrm{~s}^{-1}$.

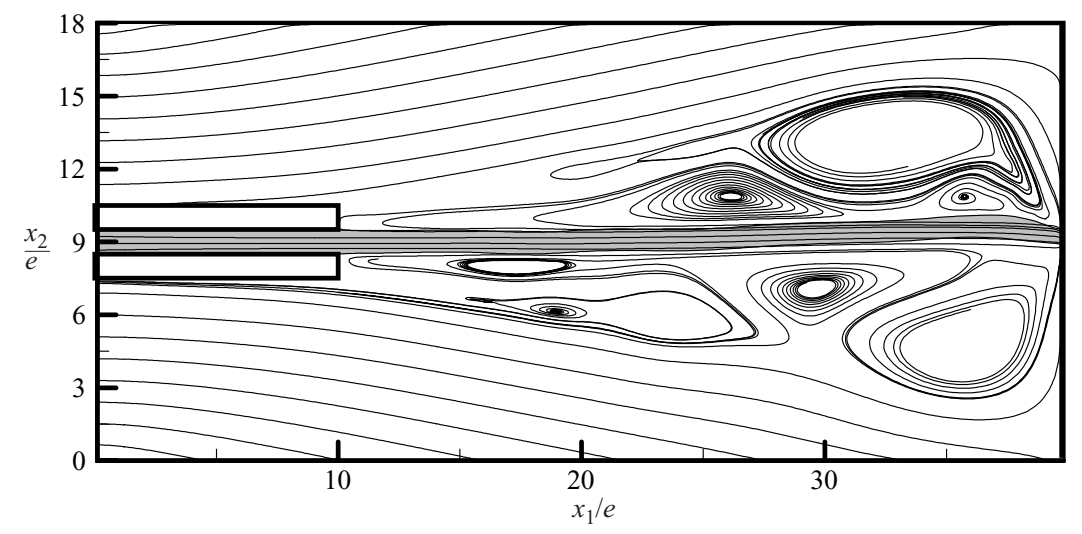

FIGURE 9. Liquid sheet location and streamlines in the gas phase for $\operatorname{Re}_{\theta_{2}}=53$ and $M=0.12$ (Case 1 of table 2).

\subsection{Influence of the gas-coflow Reynolds number}

For low gas-coflow Reynolds numbers, the configuration appears to be stable. Thus, figure 9 displays the liquid-sheet location and gas-phase streamlines at the beginning of an oscillation period for Reynolds number based on gas momentum thickness $R e_{\theta_{2}}=53$ (which yields a momentum flux ratio $M=0.12$ ). Surface tension is not considered in the model for the time being. No significant liquid-sheet deformations appear and no tendency for the sheet to fracture or break up is observed, although mild sinusoidal oscillations are apparent. Downstream of the plate trailing edges the two gaseous wakes develop a two-layer vortex structure, coupling the gas motion to that of the liquid. The pressure and viscous stress fields induced by the vortices in the gas wakes on the two sides of the liquid sheet determine the overall dynamics; the motion of the liquid sheet, in turn, influences the vortex dynamics.

The sheet dynamics change substantially as the gas-coflow Reynolds number increases. Thus, for $\operatorname{Re}_{\theta_{2}}=76(M=0.48)$, the sheet presents large deformations and a clear tendency to thinning and fracturing, as shown in figure 10 ; it is pertinent to keep in mind that the surface tension is not modelled. The influence of the liquid-sheet deformation on the vortex shedding process and on the wake vortical structures seems crucial; both from this figure and from the animated films of these computational results (López-Pagés 2000) it is apparent that the gas may posses a slow upstream 

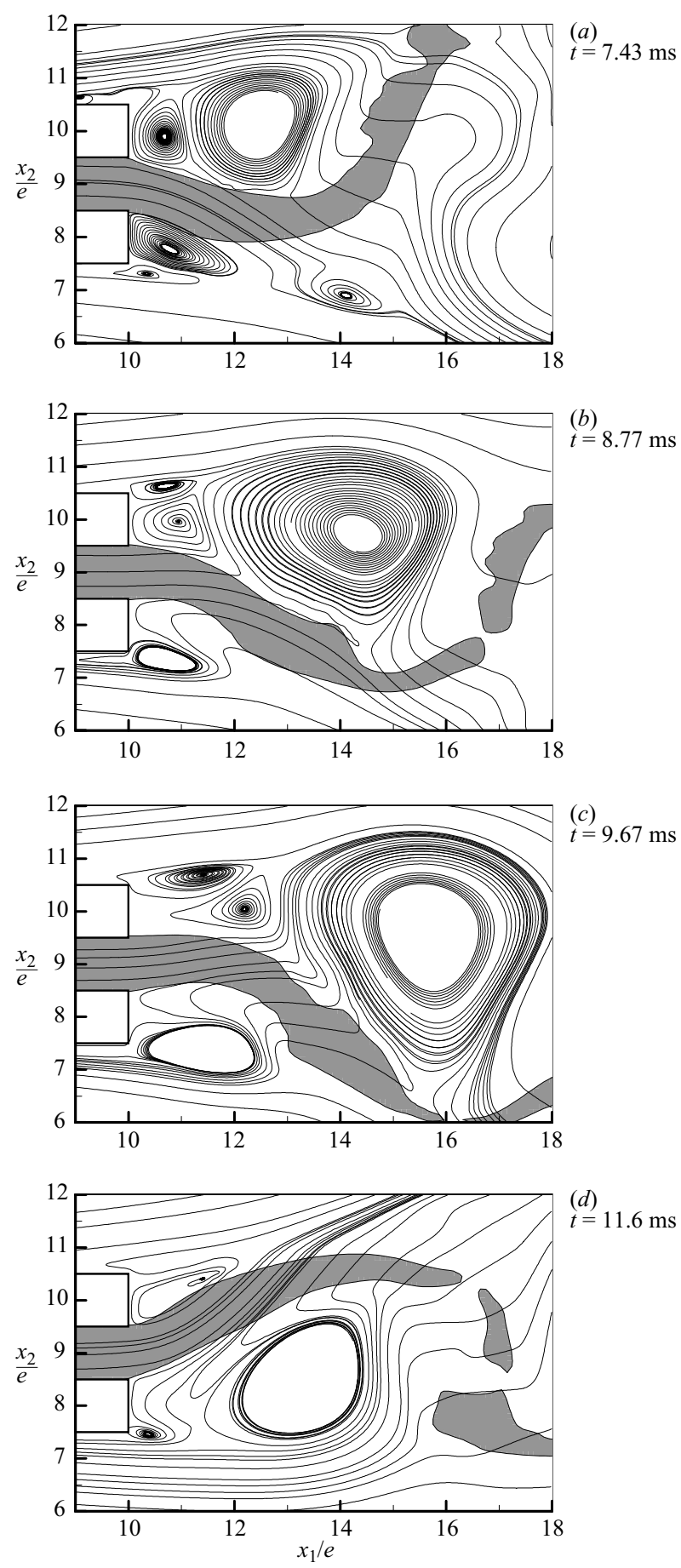

FIGURE 10. Liquid-sheet location and streamlines at four sequential instants during the liquid-sheet oscillation period for $R e_{\theta_{2}}=76$ and $M=0.48$ (Case 2). 

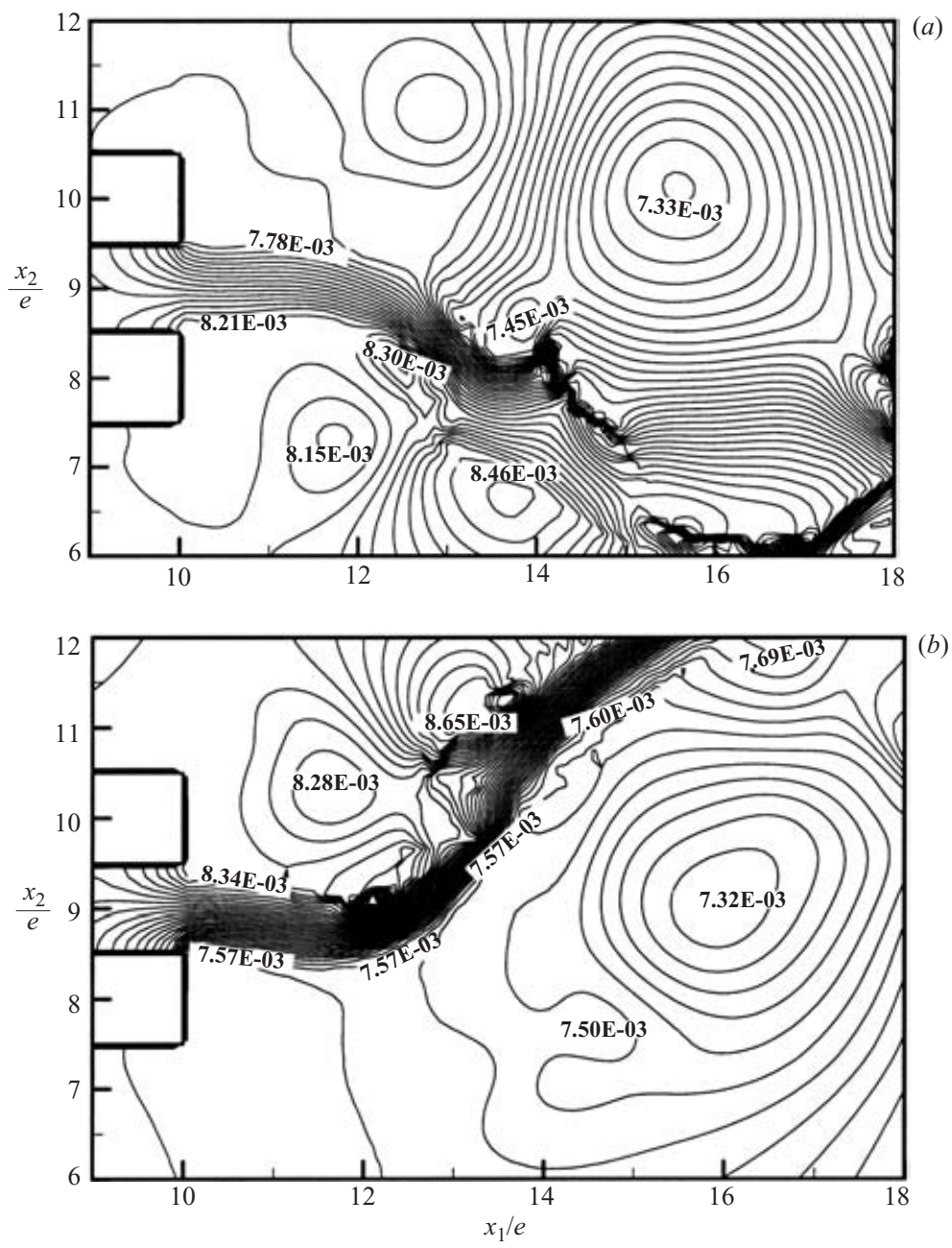

FIGURE 11. Dimensionless pressure isocontours, $p / \rho U_{2 \infty}^{2}$, corresponding to figure 10 for two instants of maximum lateral liquid-sheet displacement (Case 2).

velocity near the lower corner $(13(b)$ and $13(c))$ as the liquid sheet moves downward. The same remark applies for the upper corner of the trailing edges as the sheet flaps upwards. Figure 11 presents the isobars corresponding to the two instants of maximum lateral displacement in figure 10; the pressure differences across the two interfaces change sign, driving the sheet motion up and down alternately.

As the gas-coflow velocity is increased to yield $R e_{\theta_{2}}=92(M=1.07)$ or $R e_{\theta_{2}}=119$ $(M=2.97)$ no qualitatively significant changes are apparent. Figure 12 depicts the sheet and gas flow structure as well as the isobars for $R e_{\theta_{2}}=92$. As the dynamic pressures of the gas coflows are comparable to that of the liquid flow, as indicated by the momentum flux ratio, $M=1.07$, the sheet flapping proceeds with larger frequencies and amplitudes and the vortex shedding frequency increases. The sheet tendency to fracture (still zero surface tension) occurs closer to the injection, resulting in a smaller intact penetration length. The sheet curvatures are greater than in the previous cases 

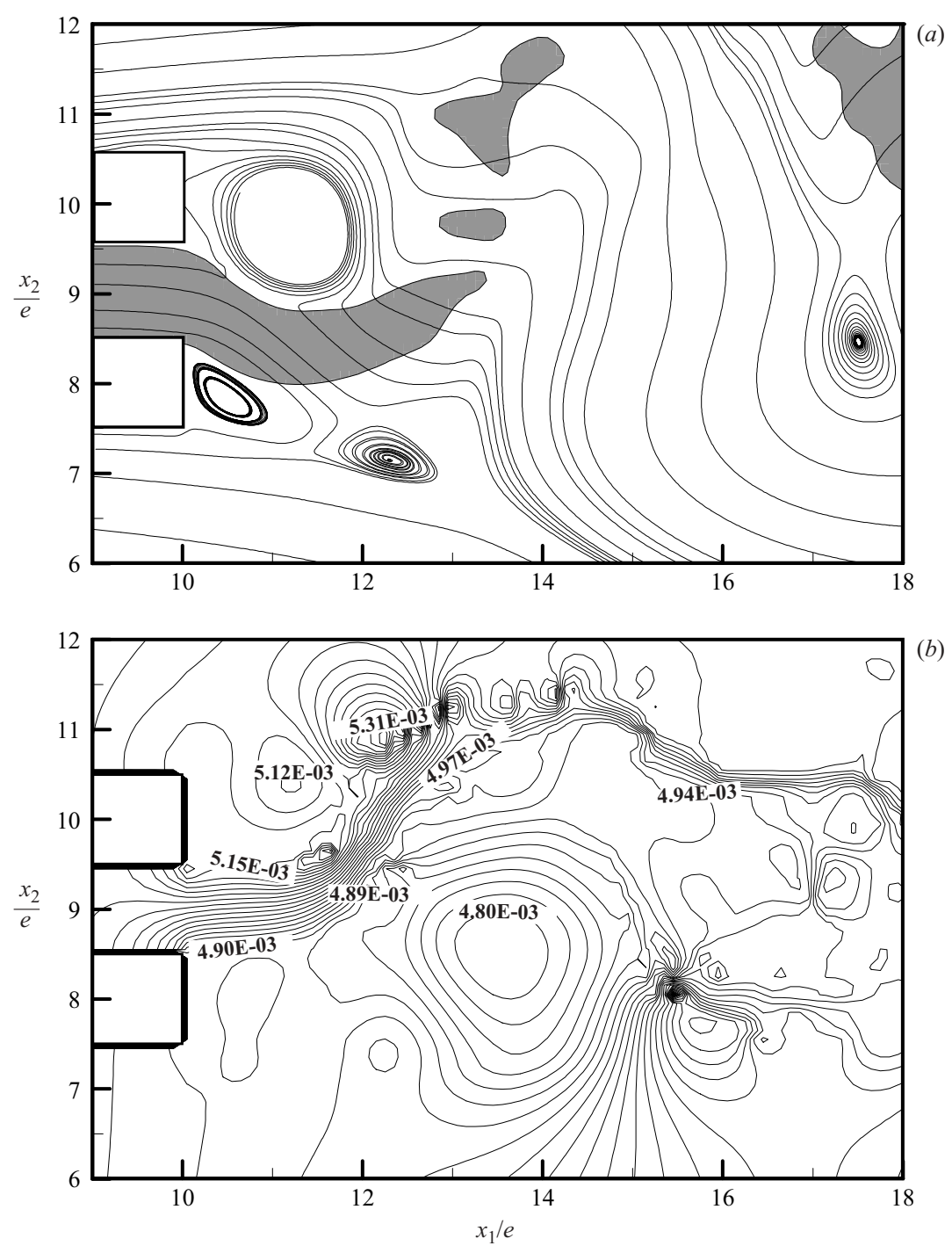

FIGURE 12. (a) Liquid-sheet location and streamlines and $(b)$ dimensionless pressure $\left(p / \rho U_{2 \infty}^{2}\right)$ isocontours, for maximum lateral liquid-sheet displacement for $\operatorname{Re}_{\theta_{2}}=92$ and $M=1.07$ (Case 3).

and the characteristic sizes of the gas vortices decrease. The pressure characteristic values are comparable to those of $R e_{\theta_{2}}=76$.

For higher gas-coflow Reynolds numbers $\left(\operatorname{Re}_{\theta_{2}}=119, M=2.97\right)$ the flow pattern is much the same except for the confining effect of the coflows upon the sheet (figure 13). This is due to the large value of the momentum flux $(M=2.97)$, which is translated into a moderate lateral sheet displacement; while for $R e_{\theta_{2}}=76$ and 92 the sheet moves laterally beyond the plates $\left(x_{2}= \pm 0.5(h+e)\right)$, for the higher $\operatorname{Re}_{\theta_{2}}=119$ the sheet amplitude is smaller. The pressure values are between one and two orders of magnitude greater than those for lower Reynolds numbers, and the sheet therefore undergoes significantly greater upward and downward accelerations. The vortex sizes in the vicinity of the nozzle are smaller than in the previous cases. 

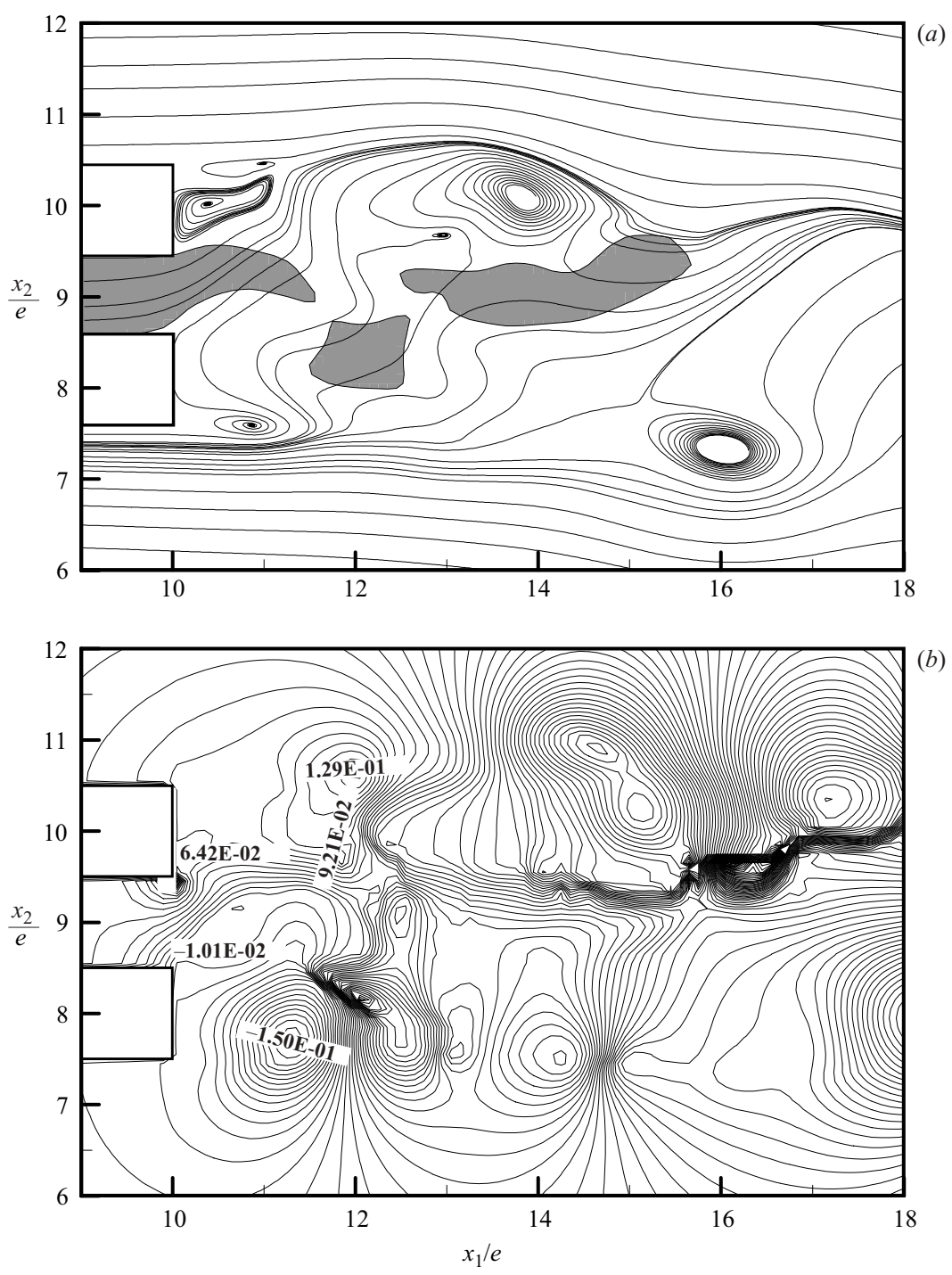

FIgURE 13. As figure 12 but for $\operatorname{Re}_{\theta_{2}}=119$ and $M=2.97$ (Case 4).

For the highest Reynolds number among those studied, $R e_{\theta_{2}}=169(M=11.89)$, the liquid sheet is significantly deformed (figure 14) and a clear tendency to fracturing is apparent just after the liquid leaves the plates; the liquid fragments detached from the sheet are violently pushed forward by the gas coflows, while they continue to be deformed and fractured. The pressure magnitudes also increase significantly compared to previous cases.

In order to quantitatively characterize the dynamics of the liquid sheet for the five gas-coflow Reynolds numbers discussed above, the wavelength $\lambda$ of the mode developing over the interface, the sheet oscillation frequency $\omega_{1}$, the gas vortex shedding frequency $\omega_{2}$, and the interface perturbation phase speed $c$, are determined. To this end, sequential images of the steady-state oscillation have been analysed and processed. In order to determine the oscillation period, two images are selected in 

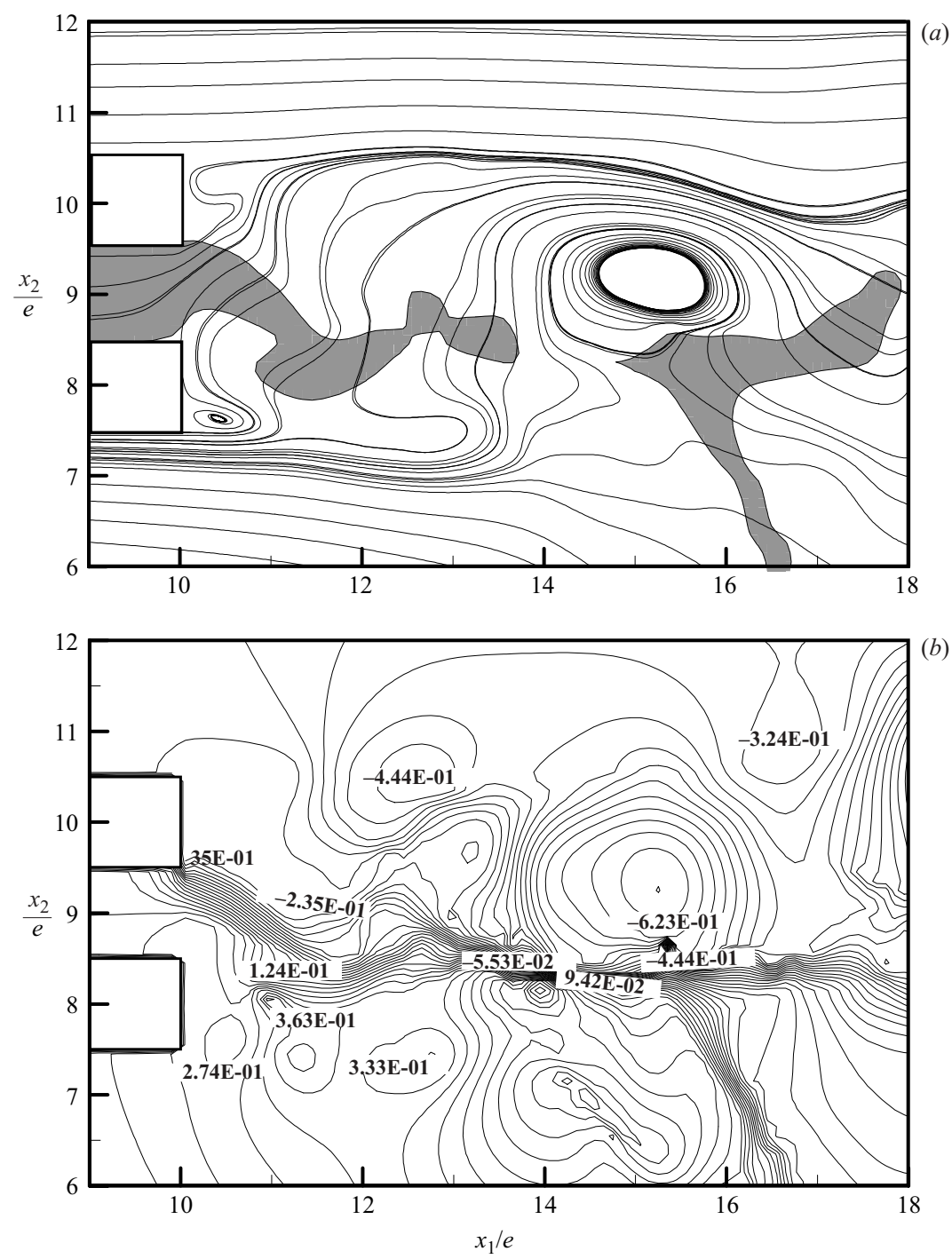

FIgURE 14. As figure 12 but for $\operatorname{Re}_{\theta_{2}}=169$ and $M=11.89$ (Case 5).

which the sheet location approximately coincides, and the time elapsed between the two pictures is established; this process is performed at the sheet exit, very near the plate trailing edges. The sheet oscillation frequency, then, results from the direct sheet/coflow interaction. The reported oscillation frequency, $\omega_{1}$, is computed from the average value of the period determined over many oscillations. The wavelength is directly measured for several images during the sheet evolution and, then, averaged over several measurements.

For $R e_{\theta_{2}}=53$, the sheet frequencies were determined at two downstream coordinates ( $x_{1}=3$ and 28 , dimensionless). The values of the frequencies at the two locations coincide. The errors in the determination of $\omega_{1}$ are associated with the time step size, $\Delta t$ and the grid cell size in the downstream direction $\left(\Delta t=10^{-3} \mathrm{~s}\right.$ for the case with $R e_{\theta_{2}}=53$ and $\Delta t=10^{-4} \mathrm{~s}$ for the cases with $R e_{\theta_{2}} \geqslant 76$ ). The relative error, $\varepsilon_{\omega_{1}}$, is of 


\begin{tabular}{crcccccc} 
Case & $R e_{\theta_{2}}$ & $\lambda(\mathrm{m})$ & $\omega_{1}(\mathrm{~Hz})$ & $\omega_{2}(\mathrm{~Hz})$ & $c\left(\mathrm{~m} \mathrm{~s}^{-1}\right)$ & \multicolumn{1}{c}{$S t_{1 e}$} & $S t_{2 e}$ \\
1 & 53 & $7.5 \times 10^{-3}$ & 170 & 823 & 0.20 & $2.7 \times 10^{-2}$ & $13.1 \times 10^{-3}$ \\
2 & 76 & $12.6 \times 10^{-3}$ & 834 & 897 & 1.67 & $13.3 \times 10^{-2}$ & $7.14 \times 10^{-3}$ \\
3 & 92 & $7.2 \times 10^{-3}$ & 1244 & 1265 & 1.42 & $19.8 \times 10^{-2}$ & $6.71 \times 10^{-3}$ \\
4 & 119 & $3.4 \times 10^{-3}$ & 2096 & 2087 & 1.13 & $33.4 \times 10^{-2}$ & $6.64 \times 10^{-3}$ \\
5 & 169 & $2.0 \times 10^{-3}$ & 3286 & 3254 & 1.05 & $52.3 \times 10^{-2}$ & $5.18 \times 10^{-3}$
\end{tabular}

TABLE 3. Characteristic parameters for the liquid-sheet oscillation and the gas vortex shedding process. Strouhal numbers for both phases are based on the plate thickness as the characteristic length.

$\begin{array}{cccrr}\text { Case } & \varepsilon_{\lambda} & \varepsilon_{\omega_{1}} & \varepsilon_{\omega_{2}} & \varepsilon_{c} \\ 1 & 1.33 & 5.40 & 13.11 & 6.73 \\ 2 & 0.79 & 1.32 & 1.43 & 2.11 \\ 3 & 1.38 & 1.98 & 2.01 & 3.36 \\ 4 & 2.94 & 3.34 & 3.32 & 6.28 \\ 5 & 5.00 & 5.47 & 5.18 & 10.47\end{array}$

TABLE 4. Relative errors for the determination of the characteristic parameters in the liquid-sheet dynamic and the gas vortex shedding frequency.

the order $\Delta t / T_{1}$, where $T_{1}$ is the specific sheet oscillation period. As $\Delta x_{1}=10^{-4} \mathrm{~m}$ for the cases studied, the relative error, $\varepsilon_{\lambda}$, in the estimation of the interfacial wavelength, $\lambda$, is of order $\Delta x_{1} / \lambda$. The relative error in the computation of the perturbation phase speed, $\varepsilon_{c}$, is of order $\varepsilon_{\omega_{1}}+\varepsilon_{\lambda}$.

The gas vortex shedding frequency behind the plate trailing edges, $\omega_{2}$, is determined from the transverse profiles of the downstream velocity component, $u_{1}\left(x_{2}\right)$. This is analysed at a short distance from the exit $\left(x_{1} / h=0.5\right)$ and two instants are identified for which the $u_{1}\left(x_{2}\right)$ profiles approximately coincide for the gas-phase region. By determining the time elapsed between the two events, the period of the vortex shedding process, $T_{2}$, is estimated and, from it, $\omega_{2}$ is calculated. Two values for the period $T_{2}$ are thus obtained and an averaged value is calculated from the different estimations. The relative error, $\varepsilon_{\omega_{2}}$, is of order $\Delta t / T_{2}$.

Tables 3 and 4 present the values of $\lambda, \omega_{1}, \omega_{2}$ and $c$, as well as the relative errors of their estimations, for the five Reynolds numbers analysed above. The wavelength $\lambda$ systematically decreases as the coflow Reynolds number increases from $R e_{\theta_{2}}=76$, while for the smallest Reynolds $R e_{\theta_{2}}=53$ it is smaller than for $R e_{\theta_{2}}=76$; the phase speed, $c$, follows a similar trend. For the flows with $R e_{\theta_{2}} \geqslant 76, c$ is not significantly different from the liquid-sheet exit velocity once the relative errors for $\lambda$ and $\omega_{1}$ are taken into account.

Figure 15 shows the variation of the Strouhal numbers of both the liquid sheet, $S t_{1 e}$, and the gas vortex shedding, $S t_{2 e}$, as functions of the momentum-flux ratio, $M$, as well as that of $S t_{2 e}$ as a function of the gas-coflow Reynolds number. Increasing $M$ causes stronger interactions among the gas coflows and the water jet, leading to larger instabilities and sheet oscillation frequencies which are reflected in the increase in the liquid Strouhal number. However, while the liquid Strouhal number increases with $M$, that for the gas follows the opposite variation, i.e. increasing $M$ through either larger gas velocities or smaller water injection speed implies a reduction in the vortex shedding frequency. On the other hand, $S t_{2 e}$ as a function of the Reynolds number 

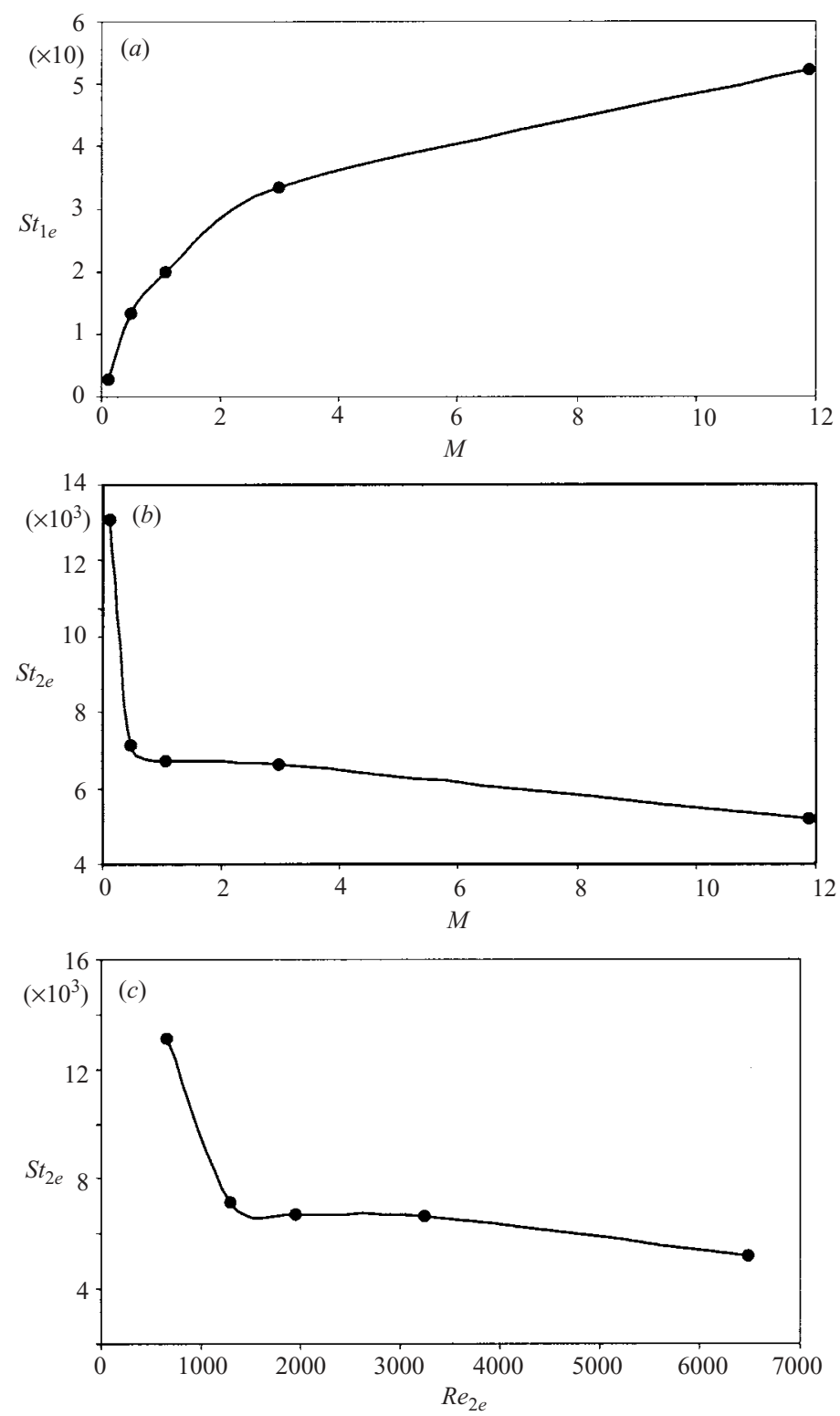

FIGURE 15. Strouhal numbers (a) $S t_{1 e}=\omega_{1} e / U_{10}$ and $(b) S t_{2 e}=\omega_{2} e / U_{2 \infty}$, as functions of momentum-flux ratio, $M=\rho_{2} U_{2 \infty}^{2} / \rho_{1} U_{1 \infty}^{2}$. (c) Strouhal number $S t_{2 e}$ as a function of the gas-coflow Reynolds-number, $\operatorname{Re}_{2 e}=e U_{2 \infty} / \nu_{2}$.

follows the common trend for single-phase wakes (Wen \& Lin 2001; Oertel 1990). This effect is explained through the presence of the liquid sheet, which obviously influences the vortex shedding process. Recall that when the liquid sheet is replaced by a gas jet the Strouhal number-Reynolds number curve (figure 7) is quite different from that in figure $15(c)$. Note that figures $15(b)$ and $15(c)$ display similar trends, as $M$ is proportional to $\left(U_{2 \infty} / U_{1 \infty}\right) R e_{2 e}$.

Figure 16 depicts the vortex shedding frequency as a function of the sheet oscillation frequency; the values of $M$ are indicated on the curves. For small values of $M$ (inertia 


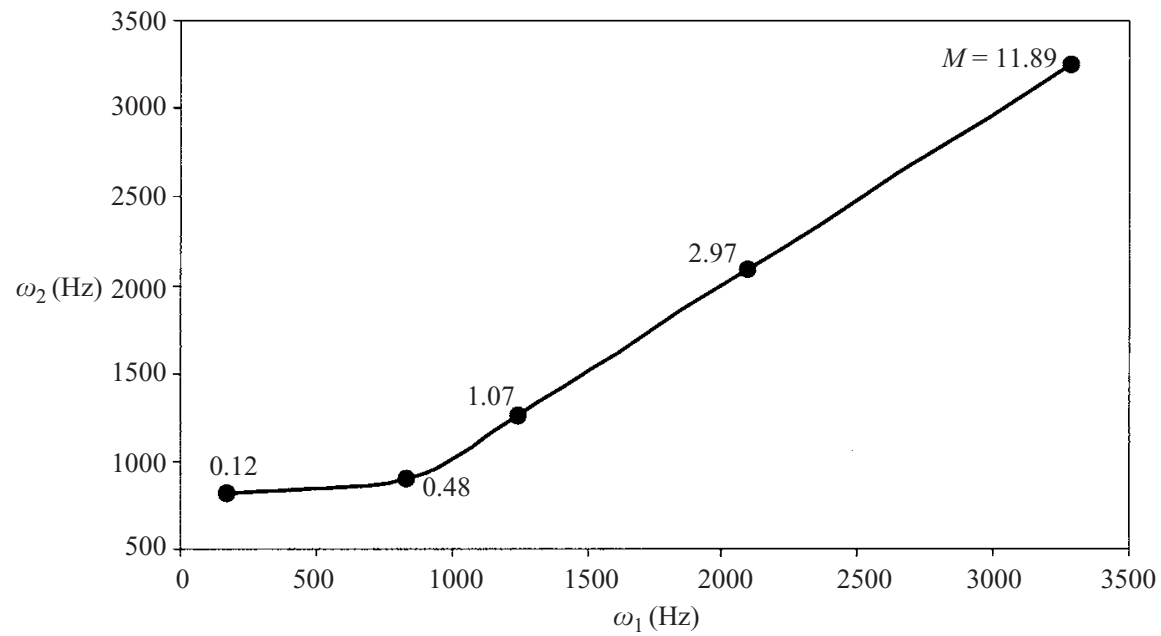

FIGURE 16. Gas-coflow vortex shedding frequency, $\omega_{2}$, as a function of the liquid sheet oscillation frequency, $\omega_{1}$.

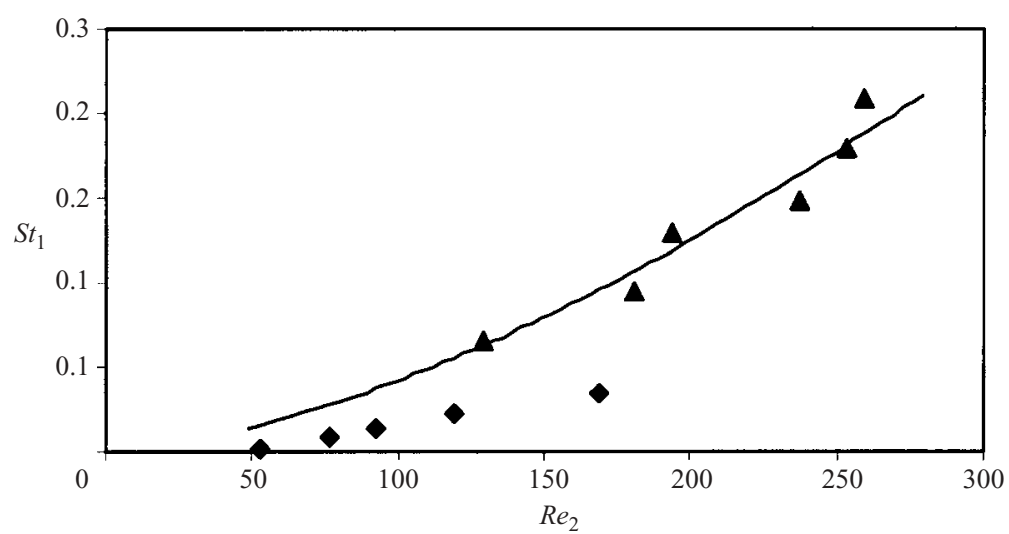

FIGURE 17. Liquid-sheet Strouhal number $S t_{1}$ (based on momentum thickness $\theta_{1}$ ), as a function of the gas-coflow Reynolds number $\operatorname{Re}_{2}$ (based on momentum thickness $\theta_{2}$ ). Diamonds correspond to numerical estimations from the present work and triangles to experimental data from Lozano et al. (2001).

forces in the gas smaller than inertia forces in the liquid or, equivalently, the gas dynamic pressure smaller than the liquid dynamic pressure), the gas streams hardly interact with the liquid sheet through weak pressure normal stresses; the gas vortex shedding frequency remains, approximately, constant while the liquid sheet oscillates with its own characteristic frequency. For $M>0.48$ or, rather, $M$ of order unity or greater, the gas vortex shedding frequency and the sheet oscillation frequency are, within numerical estimation error, almost identical. This is a new finding which uncovers, for this range of $M$, the coupling between both vortex shedding mechanisms. Such coupling offers the possibility of systematically influencing the atomization process through the modification of the gas vortex shedding pattern.

The liquid Strouhal number and dimensionless instability wavenumber, $\lambda^{*}$, as functions of the Reynolds number, $R e_{\theta_{2}}$, are presented in figures 17 and 18 , 


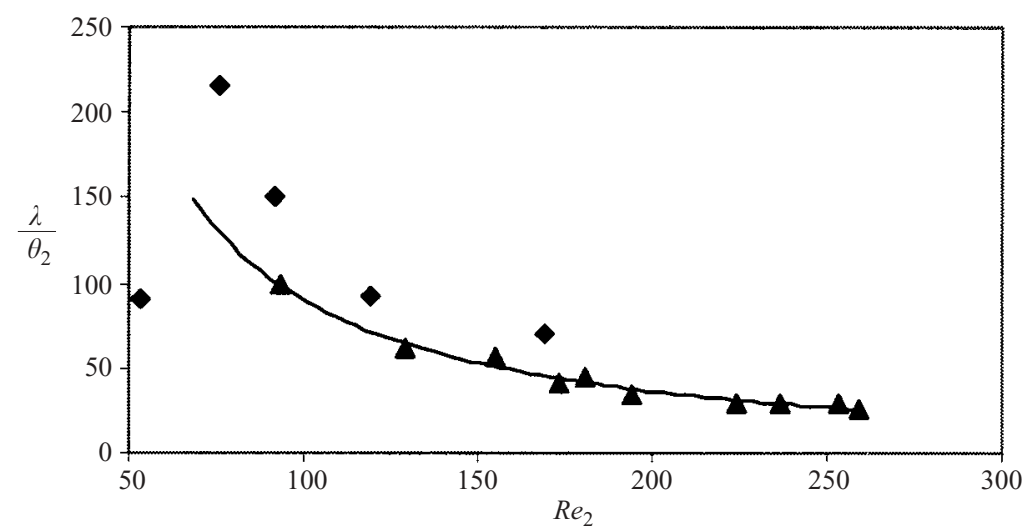

FIGURE 18. Dimensionless instability wavelength as a function of the gas Reynolds number (based on gas-flow momentum thickness $\theta_{2}$ ). Diamonds correspond to numerical estimations from the present work and triangles to experimental data from Lozano et al. (2001).

respectively; the calculated values compare reasonably well with the experimental data of Lozano et al. (2001).

\subsection{Influence of the surface tension}

A finite surface-tension coefficient $(W e=0.9)$ is now considered, while all the other parameters of the third case examined above $\left(\operatorname{Re}_{\theta_{2}}=92, M=1.07\right)$ are maintained the same. Figure 19 shows the streamlines and the sheet location at two different times, during approximately half a period of its oscillation; a distinguishing feature of this case, compared to the equivalent one with no surface tension (figure 12), is the significant increment of the liquid-sheet penetration length (intact length) into the gas coflows. Even for $M=1.07$, the stabilizing effect of the surface tension appears to prevent large sheet deformations and delays fracturing and breakup up until appropriate large local curvatures appear on the sheet interfaces. The oscillations are approximately sinusoidal. However, the sheet without and with surface tension effects oscillates with approximately the same frequency. Moreover, the perturbation wavelength with surface tension, $\lambda \approx 6.8 \times 10^{-3} \mathrm{~m}$, is not very different from that without, $\lambda \approx 7.2 \times 10^{-3} \mathrm{~m}$. Vorticity structures appear more elongated in the downstream flow direction than for the case with no surface tension, as a consequence of the smaller amplitudes of the sheet lateral motions; layers of double vortical structures are clearly present in the gas coflows. The sheet oscillation obviously modifies the gas vortex shedding process, while the pressure field induced by these vortices establishes the conditions for the sheet oscillation. A sheet oscillation frequency of $\omega_{1} \approx 1223 \mathrm{~Hz}$ is calculated which, within the previous error estimates, coincides with that of the same flow without surface tension, $\omega_{1} \approx 1244 \mathrm{~Hz}$.

\subsection{Influence of the plate thickness}

Infinitely thin plates are now considered, with flow parameters corresponding to the (finite-thickness) case with $\operatorname{Re}_{\theta_{2}}=92$ and $M=1.07$ of $\S 5.1$. The basic instability mechanisms are similar in both cases. Figure 20 displays an instant of the sheet oscillation and the corresponding pressure isocontours. The characteristic sizes of the vorticity structures are smaller than for the finite-thickness configuration (figure 12), due to the zero plate thickness; the strong and direct interaction among the liquid sheet and the two coflows leads to larger vorticity values; the latter induce pressure 

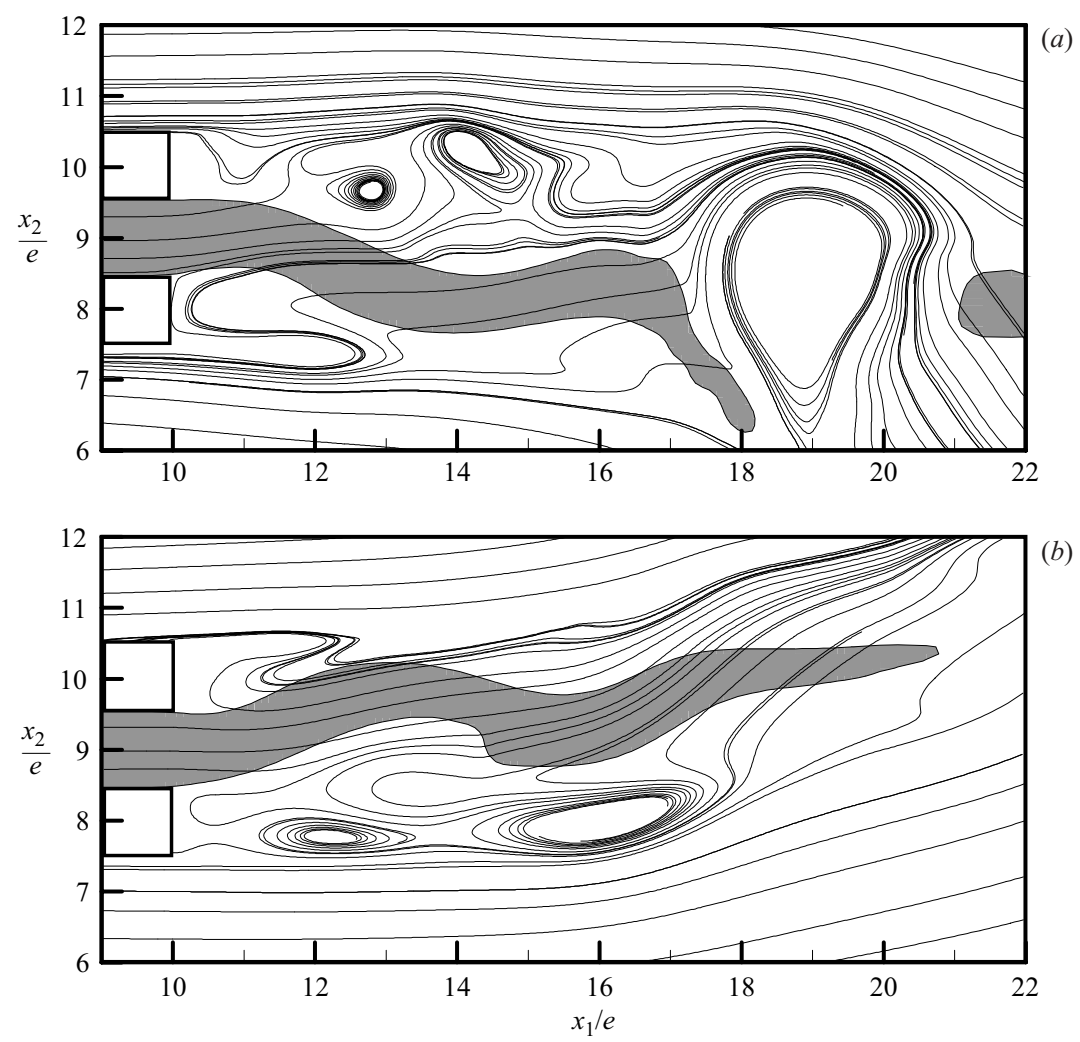

FIGURE 19. Liquid-sheet location and streamlines at two instants during the liquid-sheet oscillation period. Finite surface tension $(W e=0.9)$ for $\operatorname{Re}_{\theta_{2}}=92$ and $M=1.07$ (Case 6).

values which are two orders of magnitude greater than those for the finite-thickness situation and, consequently, there is a significant increase of the sheet oscillation frequency. The liquid sheet undergoes important deformations very near the injection section, displaying a clear tendency to rupturing (zero surface tension is considered). Table 5 summarizes the values of $\lambda, \omega_{1}$ and $c$ for finite-thickness and infinitely thin plates. The infinitesimal thickness reduces the wavelength and increases the sheetoscillation frequency yielding a phase speed not very different $(12 \%$ lower) from that for the thick-plate case.

\section{Conclusions and future work}

The two-dimensional dynamics of a flat thin liquid sheet in two parallel high-speed gas coflows has been numerically investigated very near the injection point, where the three streams merge behind the trailing edges of thin and thick solid plates.

As a frame of reference, some results have been provided with the thin liquid sheet being replaced by a gaseous jet of a density identical to that of the parallel coflows. The mixed jet/wake structure of the single-phase flow yields stream frequencies at several locations significantly greater than those for the two-phase case: for velocity ratios $U_{2 \infty} / U_{100} \approx 30$, the estimated frequencies for the former range from 1.5 to $3.1 \mathrm{kHz}$, while those for the latter are $1.265 \mathrm{kHz}$ and $1.244 \mathrm{kHz}$ for the gas vortex shedding and for the liquid-sheet oscillation frequencies, respectively. Due to the large 

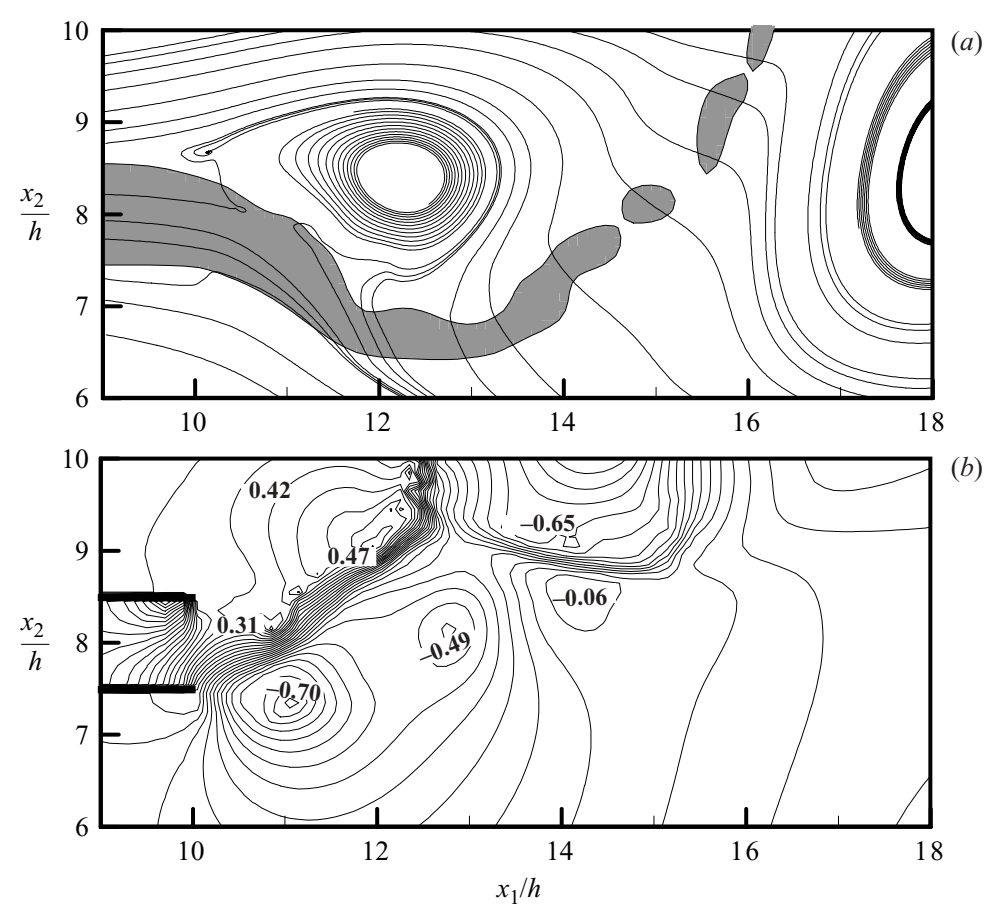

FIGURE 20. Liquid-sheet location with streamlines and the corresponding pressure isocontours at the liquid-sheet maximum lateral-displacement for $R e_{\theta_{2}}=92$ and $M=1.07$ (Case 7).

$\begin{array}{cccc}\text { Case } & \lambda(\mathrm{m}) & \omega_{1}(\mathrm{~Hz}) & c\left(\mathrm{~m} \mathrm{~s}^{-1}\right) \\ 3 & 7.2 \times 10^{-3} & 1244 & 1.42 \\ 7 & 3.4 \times 10^{-3} & 2307 & 1.25\end{array}$

TABLE 5. Characteristic parameters of the liquid sheet oscillation for Cases 3 (thick plate trailing edges) and 7 (infinitely thin plate trailing edges).

sheet inertia $\left(\rho_{1} / \rho_{2} \approx 10^{3}\right)$, the flow oscillations are slowed down in the presence of the liquid. At the same time, the vortex-shedding process is substantially modified by the liquid-sheet flapping motion; some upstream inverse flow may take place near the upper and lower corners of the thick solid plates, coinciding with upward or downward motion of the sheet, respectively.

The Strouhal number as a function of the Reynolds number for single-phase flow (figure 7) follows the usual trend for wakes for $R e_{e} \leqslant 1300$. The reduction can be attributed to the mixed jet/wake nature of the flow; the presence of the jet reduces the wake vortex-shedding frequencies. On the other hand the gas Strouhal number monotonically decreases as the Reynolds number increases (figure 15c), due to the influence of the sheet motion on the gas vortex-shedding process. Values of the Strouhal number vary from order $10^{-4}$ for the former case to $10^{-2}$ for the latter.

The pressure and strain fields induced by the vorticity patterns in the gaseous streams are responsible for the liquid-sheet dynamics. As the gas Reynolds number increases the vortical flow structures are modified; their tendency to grow laterally is balanced by the action of the confining gas flow, which also elongates the vortices in 
the downstream direction. In parallel, the dimensionless isobars span over six orders of magnitude, increasing with the Reynolds number; this translates into an increment of the sheet oscillation frequency, $\omega_{1}$, and reduction of the perturbation wavelenght, $\lambda$, as the Reynolds number increases. Computed and experimentally determined values of $\omega_{1}$ and $\lambda$ compare reasonably well.

Estimated gas vortex-shedding frequencies are, within numerical determination error, identical to liquid-sheet oscillation frequencies for momentum flux ratios, $M$, greater than unity. This is interpreted in terms of the dynamic pressures of the gas and liquid flows; when the former is equal to or greater than the latter, the gas and liquid dynamics are perfectly coupled. For $M<1.0$ the vortex-shedding process and the sheet oscillation proceed independently.

Considering surface tension $(W e=0.9)$ does not significantly alter the instability development process. The vortices are more elongated in the downstream direction compared to the case with no surface tension $(W e=\infty)$. The stabilizing effect of the surface tension is also apparent from larger intact sheet lengths.

The next obvious extension of this work is to address the three-dimensional case. The influence of the liquid/gas density ratio on the flow dynamics will also be explored; while some existing, and otherwise successful, codes are limited to values less than 10, the present algorithm seems to be able to handle values as large as $10^{3}$ with no problem. The case of zero gas velocity, $U_{2 \infty}=0$, of relevance to pressure atomizers, will also be taken up.

The present paper is a part of E. López-Pagés' PhD Dissertation, who gratefully acknowledges the support under an AECI (Spanish International Cooperation Agency) Fellowship. Drs A. Lozano and F. Barreras provided some helpful comments on the original manuscript.

\section{REFERENCES}

Ashgriz, N. \& Poo, J. Y. 1991 FLAIR: Flux line-segment model for advection and interface reconstruction. J. Comp. Physut. 93, 449-468.

BARLEY, J. J. 1988 A survey of operator splitting applied to upwind differencing. Numerical Analysis Report 2/88. Dept. of Mathematics, University of Reading.

BARRERAS, F. 1998 Experimental study of the break-up and atomization of a liquid sheet. PhD Dissertation, University of Zaragoza (in Spanish).

Berthoumieu, P. \& CARENTZ, H. 2000 Experimental study of a thin planar liquid sheet disintegration. Eighth Intl Conf. Liquid Atomization and Spray Systems, Pasadena, CA (ed. L. Fuchs), pp. 1120-1126.

Brackbill, J. U., Kothe, D. B. \& Zemach, C. 1992 A continuum method for modelling surface tension. J. Comput. Phys. 100, 335-354.

Chen, G., Krarif C., Zaleski S. \& Li, J. 1999 Two-dimensional Navier-Stokes simulation of breaking waves. Phys. Fluids 11, 121-133.

Clark, C. J. \& Dombrowski, N. 1972 Aerodynamic instability and disintegration of inviscid liquid sheets. Proc. R. Soc. Lond. A 329, 467-478.

Cousin, J. \& Dumouchel, C. 1996 Effect of viscosity on linear instability of a flat liquid sheet. Atomization Spray 6, 563-576.

Crapper, G. D., Dombrowski, N. \& Jepson, W. P. 1975 Waves on thin sheets of non-newtonian liquids. Proc. R. Soc. Lond. A 342, 225-236.

Cristini, V., BLAWZDZIEWICS, J. \& LowENBERG, M. 1998 Drop breakup in three-dimensional viscous flows. Phys. Fluids 110, 8, 1781-1783.

FritTs, M. J. \& BoRIS, J. P. 1979 The Lagrangian solution of transient problems in hydrodynamics using a triangular mesh. J. Comput. Phys. 31, 173-215. 
Gaskell, P. H. \& LAU, A. K. C. 1988 Curvature compensated convective transport: SMART a new boundedness-preserving transport algorithm. Intl J. Numer. Meth. Fluids 8, 617-641.

Glimm, J., Marchesin, D. \& McBryan, O. 1981 A numerical method for two phase flow with unstable interface. J. Comput. Phys. 39, 179-200.

Hagerty, W. W. \& SheA, J. F. 1955 A study of the stability of plane fluid sheets. Trans. ASME: J. Appl. Mech. 22, 509-514.

Harten, A. \& Hyman, J. M. 1983 A self-adjusting grid for the computation of weak solutions of hyperbolic conservation laws. J. Comput. Phys. 50, 235-269.

Hauke, G., Dopazo, C., Barreras, F., Lozano. A. \& Hernandez, A. 2001 Linear stability analysis of a viscous liquid sheet in coflow with a viscous gas. Flow, Turbulence Combust. 67, 235-265.

Hirsch, C. 1988 Numerical Computation of Internal and External Flows. Wiley Interscience.

Hirt, C. W., Amsdem, A. A. \& CoOK, J. L. 1974 An arbitrary lagrangean-eulerian computing methods for all speeds. J. Comput. Phys. 14, 227-253.

Hirt, C. W. \& Nichols, B. D. 1981 Volume of Fluid (VOF) method for the dynamics of free boundaries. J. Comput. Phys. 39, 201-225.

Hyman, J. M. 1983 Adaptive moving mesh methods for partial differential equations. Advances in reactor computations. Am. Nucl. Rep. 24-43.

Hyman, J. M. 1984 Numerical methods for tracking interfaces. Physica 12D, 396-407.

Ibrahim, E. A. 1994 Spatial instability of a viscous liquid sheet. AIAA Paper 94-0562, pp. 1-7.

IBRAHIM, E. A. 1995 Effects of compressibility on the instability of liquid sheets. AIAA Paper 95-2431.

Ibrahim, E. A. \& Akpan, E. T. 1996 Three-dimensional instability of viscous liquid sheets. Atomization Sprays 6, 649-665.

JAZAYERI, S. A. \& LI, X. 2000 Nonlinear instability of plane liquid sheets. J. Fluid Mech. 406, 281-308.

Kim, I. \& Sirignano, W. A. 2000 Three-dimensional wave distortion and disintegration of thin planar liquid sheets. J. Fluid Mech. 410, 147-183.

Lafaurie, B., Nardone, C., Scardovelli, R., Zaleski, S. \& Zanetti, G. 1994 Modelling merging and fragmentation on multiphase flows with SURFER. J. Comput. Phys. 113, 134-147.

Lasheras, J. C. \& ChOI, H. 1988 Three-dimensional instability of a plane free shear layer: an experimental study of the formation and evolution of streamwise vortices. J. Fluid Mech. 189, 53-86.

Lasheras, J. C. \& Hopfinger, E. J. 2000 Liquid jet instability and atomization in a coaxial gas stream. Annu. Rev. Fluid Mech. 32, 275-308.

Lasheras, J. C., Villermaux, E. \& Hopfinger, E. 1998 Breakup and atomization of a round water jet by a high speed annular air jet. J. Fluid Mech. 35, 351-379.

VAN LEER, B. 1979 Towards the ultimate conservative difference scheme. V. A second-order sequel to Godunov's method. J. Comput. Phys. 32, 101-136.

LI, X. 1994 On the instability of plane liquid sheets in two gas streams of unequal velocities. Acta Mechanica 106, 137-156.

LI, X. \& TANKIN, R. S. 1991 On the temporal instability of a two-dimensional viscous liquid sheet. J. Fluid Mech. 226, 425-443.

Lin, S. P., Lian, Z. W. \& Creighton, B. J. 1990 Absolute and convective instability of a liquid sheet. J. Fluid Mech. 220, 673-689.

LÓPEZ-PAGÉs, E. 2000 Numerical simulation of instabilities on liquid/gas interfaces. PhD Dissertation, University of Zaragoza (in Spanish).

LöTSTEDT, P. 1982 A front tracking method applied to Burgers'equation and two-phase porous flow. J. Comput. Phys. 47, 211-228.

Lozano, A., Barreras, F. Hauke, G. \& Dopazo, C. 2001 On the longitudinal perturbation of an air-blasted liquid sheet. J. Fluid Mech. 437, 143-173.

Lozano, A., Call, C. J., Dopazo, C. \& García-Olivares, A. 1996 An experimental and numerical study of the atomization of a planar liquid sheet. Atomization Sprays 6, 77-94.

Lozano, A., García-Olivares, A. \& Dopazo, C. 1998 The instability growth leading to a liquid sheet. Phys. Fluids 10, 2188-2197.

Mansour, A. \& Chigier, N. 1990 Disintegration of liquid sheets. Phys. Fluids A 2, 706-719.

Mansour, A. \& Chigier, N. 1991 Dynamic Behavior of Liquid Sheets. Phys. Fluids A 3, 2971-2980. 
Mashayek, F. \& Ashgriz, N. 1995 A hybrid finite-element-volume-of-fluid method for simulating free surface flows and interfaces. Intl J. Numer. Meth. Fluids 20, 1363-1380.

Meiburg, E. \& Lasheras, J. C. 1988 Experimental and numerical investigation of the threedimensional transition in plane wakes. J. Fluid Mech. 190, 1-37.

Nichols, B. D. \& HiRT, C. W. 1975 Methods for calculating multi-dimensional, transient free surface flows past bodies. Proc. First Intl Conf. Numer. Ship Hydrodynamics, Gaithersburg.

Oertel Jr., H. 1990 Wakes behind bluff bodies. Annu. Rev. Fluid Mech. 22, 539-564.

Patankar, S. V. 1980 Numerical Heat Transfer and Fluid Flow. Hemisphere.

Ramshaw, J. D. \& Trapp, J. A. 1976 A numerical technique for low-speed homogeneous two-phase flow with sharp interfaces. J. Comput. Phys. 21, 438.

Rangel, R. H. \& SiRignano, W. A. 1991 The linear and non-linear shear instability of a fluid sheet. Phys. Fluids A 3, 2392-2400.

RAYNAL, L. 1997 Instability and entrainment at the interface of a liquid-gas mixing layer. PhD Dissertation, University Joseph Fourier - Grenoble I (in French).

Ryskin, G. \& Leal, J. G. 1984 Numerical solution of free-boundary problems in fluid mechanics. J. Fluid Mech. 148, 1-43.

Savart, F. 1833 Memoire sur le choc d'une veine liquide lancée contre un plan circulaire. Ann. Chim. 54, 56-87.

SCARDOVELLI, R. \& ZALESKI, S. 1999 Direct numerical simulation of free-surface and interfacial flow. Annu. Rev. Fluid Mech. 31, 567-603.

Sмith, F. T. 1982 On the high Reynolds number theory of laminar flows. IMA J. Appl. Maths 28, 207-281.

SQuire, H. B. 1953 Investigation of the instability of moving liquid film. Brit. J. Appl. Phys. 4, $167-169$.

Stapper, B. E. \& Samuelsen, G. S. 1990 An experimental study of the breakup of a two-dimensional liquid sheet in the presence of co-flow air shear. AIAA Paper 89-0461.

Stapper, B. E., Sowa, W. A. \& Samuelsen, G. S. 1992 An experimental study of the effects of liquid properties on the breakup of a two-dimensional liquid sheet. J. Engng for Gas Turbine 114, $39-45$.

Stewartson, K. 1969 On the flow near a trailing edge of a flat plate. Proc. R. Soc. Lond. A 306, 275-290.

Stone, H. L. 1968 Iterative solution of implicit approximations of multidimensional partial differential equations. SIAM J. Numer. Anal. 5, 530-558.

TAYLOR, G. I. 1959 The dynamics of thin sheets of fluid I. Water bells. Proc. R. Soc. Lond. A 253, 280-296.

Teng, C. H., Lin, S. P. \& Chen, J. N. 1997 Absolute and convective instability of a viscous liquid curtain in a viscous gas. J. Fluid Mech. 332, 105-120.

Vich, G., Dumouchel, C. \& Ledoux, M. 1996 Mechanisms of disintegration of flat liquid sheets. 12th Ann. Conf. ILASS-Europe, Lund, Sweden, June 19-21.

Waterson, N. P. \& DeCONNICK, H. 1994 A unified approach to the design and application of bounded high-order convection schemes. Proc. Numer. Meth. Laminar Turb. Flow 9, 203-214.

Wen, C.-Y. \& LIN, C.-Y. 2001 Two-dimensional vortex shedding of a circular cylinder. Phys. Fluids 13, 557-560.

Welch, J. E., Harlow, F. H., Shannon, J. P. \& Daly, B. J. 1966 The MAC method: A computing technique for solving viscous incompressible transient fluid flow problems involving free surfaces. Los Alamos Scientific Lab. Rep. LA-3425.

YANenKo, N. N. 1971 The Method of Fractional Steps. Springer.

YANG, H. Q. 1992 Interfacial instability between a liquid sheet and the surrounding compressible gas. AIAA Paper 92-0461.

York, J. L., StubBs, H. E. \& TeK, M. R. 1953 The mechanisms of disintegration of liquid sheets. Trans. ASME, October, $1279-1286$

ZALESKI, S. 1996 Simulation of high Reynolds number breakup of liquid-gas interfaces in Combustion and turbulence in two-phase flows. Lecture Series 1996-02. Von Karman Institute of Fluid Dynamics. 